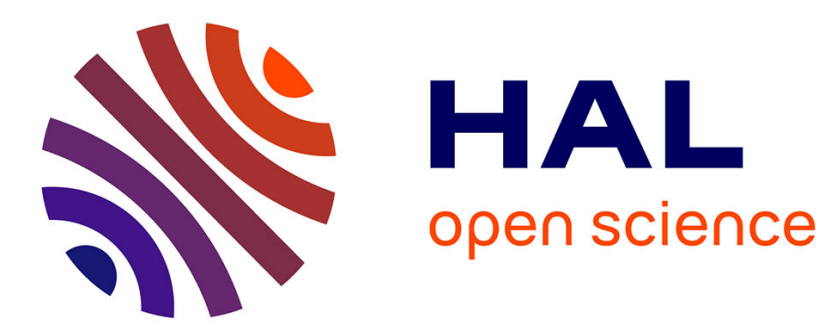

\title{
A Cellular Howe Theorem
}

\author{
Peio Borthelle, Tom Hirschowitz, Ambroise Lafont
}

\section{To cite this version:}

Peio Borthelle, Tom Hirschowitz, Ambroise Lafont. A Cellular Howe Theorem. LICS '20: 35th Annual ACM/IEEE Symposium on Logic in Computer Science, Jul 2020, Saarbrücken, Germany. pp.273-286, 10.1145/3373718.3394738 . hal-02880876

\section{HAL Id: hal-02880876 https://hal.science/hal-02880876}

Submitted on 25 Jun 2020

HAL is a multi-disciplinary open access archive for the deposit and dissemination of scientific research documents, whether they are published or not. The documents may come from teaching and research institutions in France or abroad, or from public or private research centers.
L'archive ouverte pluridisciplinaire HAL, est destinée au dépôt et à la diffusion de documents scientifiques de niveau recherche, publiés ou non, émanant des établissements d'enseignement et de recherche français ou étrangers, des laboratoires publics ou privés. 


\section{A Cellular Howe Theorem}

\author{
Peio Borthelle \\ Univ. Grenoble Alpes, Univ. Savoie \\ Mont Blanc, CNRS, LAMA \\ 73000, Chambéry, France
}

\author{
Tom Hirschowitz \\ Univ. Grenoble Alpes, Univ. Savoie \\ Mont Blanc, CNRS, LAMA \\ 73000, Chambéry, France
}

\author{
Ambroise Lafont \\ Gallinette, Inria Nantes, IMT \\ Atlantique \\ Nantes, France
}

\begin{abstract}
We introduce a categorical framework for operational semantics, in which we define substitution-closed bisimilarity, an abstract analogue of the open extension of Abramsky's applicative bisimilarity. We furthermore prove a congruence theorem for substitution-closed bisimilarity, following Howe's method. We finally demonstrate that the framework covers the call-by-name and call-by-value variants of $\lambda$-calculus in big-step style. As an intermediate result, we generalise the standard framework of Fiore et al. for syntax with variable binding to the skew-monoidal case.
\end{abstract}

CCS Concepts: • Theory of computation $\rightarrow$ Semantics and reasoning; Categorical semantics; Operational semantics.

Keywords: operational semantics, category theory, bisimilarity, congruence, Howe's method

\section{ACM Reference Format:}

Peio Borthelle, Tom Hirschowitz, and Ambroise Lafont. 2020. A Cellular Howe Theorem. In Proceedings of the 35th Annual ACM/IEEE Symposium on Logic in Computer Science (LICS '20), Fuly 8-11, 2020, Saarbrücken, Germany. ACM, New York, NY, USA, 27 pages. https: //doi.org/10.1145/3373718.3394738

\section{Introduction}

\subsection{Motivation}

In research on programming language design and implementation, ideas are often presented on one, simple example. E.g., abstract interpretation, separation logic, or gradual typing were all presented on a single language, and later adapted to other settings. Usually, the presented ideas are thought of as widely applicable and their scope is clear to the experts, but no attempt is made at delimiting it precisely and formally. As a consequence, these ideas cannot be freely

Permission to make digital or hard copies of all or part of this work for personal or classroom use is granted without fee provided that copies are not made or distributed for profit or commercial advantage and that copies bear this notice and the full citation on the first page. Copyrights for components of this work owned by others than ACM must be honored. Abstracting with credit is permitted. To copy otherwise, or republish, to post on servers or to redistribute to lists, requires prior specific permission and/or a fee. Request permissions from permissions@acm.org.

LICS '20, fuly 8-11, 2020, Saarbrücken, Germany

() 2020 Association for Computing Machinery.

ACM ISBN 978-1-4503-7104-9/20/07...\$15.00

https://doi.org/10.1145/3373718.3394738 reused, even in slightly different contexts, they always have to be adapted, and reproved.

We think the reason for this is that appropriate mathematical concepts are missing: there is no widely accepted notion of programming language, so that we cannot state properties like "for all programming languages of such shape, the following idea works". Such a general notion should account for both

(i) the interaction between syntax and dynamics, as involved in, e.g., structural operational semantics [32], or in statements or proofs of results like type soundness, congruence of program equivalence, or compiler correctness, and

(ii) denotational semantics, in the sense of including not only operational, syntactic models but also others, typically ones in which program equivalence is coarser.

\subsection{Context}

In recent work [21], Hirschowitz proposed a new abstract approach to operational semantics, and demonstrated its expressive power by proving abstract versions of classic results in process algebra, including for the first time an abstract soundness result for bisimulation up to context in the presence of variable binding. Bisimulation up to context is an efficient technique [33] for proving program equivalences, which had previously been proved correct at a similar level of generality [7], but only without binding.

Briefly, in the new setting, a language equipped with a collection of operational semantics rules is viewed as a monad $\mathscr{T}$ on a transition category $\mathscr{C}$, typically a category of labelled graphs. The idea is that, on vertices, $\mathscr{T}$ defines the syntax of the considered language: for any $X \in \mathscr{C}$, the vertices of $\mathscr{T}(X)$ are terms with free variables in vertices of $X$. Similarly, transitions in $\mathscr{T}(X)$ are derivation trees following the given rules, with axioms in transitions of $X$. So in particular $\mathscr{T}(0)$ is precisely the syntactic transition system.

Now, properties like congruence of bisimilarity or soundness of bisimulation up to context may be expressed in this setting, and their proofs in [21] rely on two crucial properties of $\mathscr{T}$.

- First, it is familial $[9,12]$, and even cellular in a sense close to Garner and Hirschowitz [18]. Familiality yields abstract analogues of syntactic notions like contexts and partial derivation trees, and cellularity enforces well-formedness conditions on the collection of premises of each transition rule, very roughly the fact 
that the premises of any transition $f\left(x_{1}, \ldots, x_{n}\right) \rightarrow x^{\prime}$ consist of transitions from $x_{1}, \ldots, x_{n}$.

- Furthermore, in order to prove, e.g., congruence of bisimilarity for a free algebra $\mathscr{T}(X)$, the second property that we need is that the monad multiplication $\mu_{X}: \mathscr{T}^{2}(X) \rightarrow \mathscr{T}(X)$ is a functional bisimulation. Let us call this compositionality of $\mathscr{T}$ at $X$.

\subsection{Overview}

In this paper, we extend the approach to higher-order languages, taking as a running example the call-by-name, big step $\lambda$-calculus, equipped with the so-called open extension of Abramsky's applicative bisimilarity [2], which we here call substitution-closed bisimilarity.

Higher-order languages challenge the approach of [21] notably because transition rules rely on proper substitution, as opposed to mere renaming.

Indeed, if we follow one Fiore et al.'s categorical framework $[13-15,17,19]$ for syntax with substitution in the presence of variable binding, the monad $\mathscr{T}$ we obtain is not familial. Informally, in order to model substitution, $\mathscr{T}$ features a form of explicit substitution [1], which turns out to be too flexible for familiality to work. Let us explain this in a bit more detail. In the case of pure $\lambda$-calculus, the set of terms is viewed as indexed over (finite) sets of free variables, and natively equipped with renaming operations. I.e., for any map $f: m \rightarrow n$ between finite ordinals, we get a map $X(f): X(m) \rightarrow X(n)$ from terms with $m$ free variables to terms with $n$ free variables. Substitution is modelled as a map $X \otimes X \rightarrow X$, where elements of $(X \otimes X)(m)$ are 'explicit' substitutions $e\left(e_{1}, \ldots, e_{n}\right)$, with $e \in X(n)$ and $e_{1}, \ldots, e_{n} \in X(m)$. The problem is that such explicit substitutions are standardly considered equivalent modulo simple relations, e.g.,

$$
(X(\operatorname{swap})(e))\left(e_{1}, e_{2}\right)=e\left(e_{2}, e_{1}\right),
$$

where swap: $2 \rightarrow 2$ swaps the two given elements.

It is precisely because of this quotienting that the obtained monad is not familial. In order to restore familiality and thus be able to follow the approach of [21], we switch to a more rigid notion of explicit substitution, which in fact forces us to change both the ambient category in examples and the general structure. The standard category for untyped calculi is the functor category [Set ${ }_{f}$, Set] of covariant presheaves on a skeleton of finite sets, with its standard monoidal structure. We now need to switch to mere $\mathbb{N}$-indexed families of sets, on which the relevant tensor product only yields skew-monoidal structure [5, 37]. Skew-monoidal structure is a weakening of plain monoidal structure, in which structural associativity and unitality isomorphisms may not be invertible.

As a final twist on this, syntax is standardly specified by a so-called pointed-strong endofunctor on the considered monoidal category, typically [Set ${ }_{f}$, Set], but the analogous endofunctor on $[\mathbb{N}$, Set $]$ is not pointed strong. We thus need to resort to a weaker notion which we call structurally strong.

In summary, and this is our first contribution, we generalise the standard framework of pointed strong endofunctors on a monoidal category, to what we call structurally strong endofunctors $\Sigma_{0}$ on a skew-monoidal category. We characterise the syntax as the initial $\Sigma_{0}$-monoid, i.e., $\Sigma_{0}$-algebra with the syntax as the initial $\Sigma_{0}$-monoid, i.e., $\Sigma_{0}$-algebra with compatible monoid structure (Theorem 2.15), a characterisation we mechanically verify in Coq (see supplementary material), relying on the UniMath library [39]. Finally, we prove that the obtained monad $\mathscr{T}_{0}$ is familial, along with the fact that the natural transformation $\Sigma_{0} \rightarrow \mathscr{T}_{0}$ preserves familiality, in a suitable sense (Theorem 2.19).

We thus obtain a framework for syntax with substitution in the presence of variable binding which lends itself to the familial/cellular approach of [21]. The next step is to model the dynamics. We first introduce transition $\Sigma_{0}$-monoids, which are intuitively transition systems whose vertices are equipped with $\Sigma_{0}$-monoid structure. Then, adapting ideas from Fiore [17] and Ahrens et al. [4, 20] to the cellular approach, we consider transition rules specified by a syntactically free endofunctor $\Sigma_{1}$ on transition $\Sigma_{0}$-monoids, models being given by a special kind of algebras, called vertical. Under finitarity hypotheses, as our second contribution, we characterise the syntactic transition system as the initial vertical algebra (Theorem 4.20).

Finally, we define substitution-closed bisimilarity, which in examples instantiates to the open extension of applicative bisimilarity. Our goal is thus to prove that substitutionclosed bisimilarity is a congruence. However, we meet a last significant difficulty, namely that because of explicit substitution, compositionality fails. In fact, a slightly weaker property holds, essentially compositionality w.r.t. operations from $\Sigma_{0}$ (as opposed to explicit substitution). As a third and final contribution, under an additional cellularity hypothesis for $\Sigma_{1}$, we follow Howe's construction prove abstractly that substitution-closed bisimilarity is a congruence (Theorem 5.19). We show that the result applies to call-by-value and call-by-name variants of big-step, pure $\lambda$-calculus.

Altogether, under suitable hypotheses, our contributions provide a systematic construction, from the basic $\Sigma_{0}$ and $\Sigma_{1}$, of a syntactic transition system whose substitution-closed bisimilarity is a congruence.

\subsection{Related work}

The main framework meeting the above criteria ( $i$ ) and ( $i i)$ is bialgebraic semantics [38], including a few variants [11, 36]. As far as we know, these approaches do not cover higherorder languages like the $\lambda$-calculus, which was one of the motivations for our work. Among more recent work, quite some inspiration was drawn from Ahrens et al. [4, 20], notably in the use of vertical algebras. However, a difference is 
that we do not insist that transitions be stable under substitution. Links with other relevant work, e.g., Bodin et al. [6], though desirable, remain unclear, perhaps because of the very different methods used. Furthermore, the cellularity used here is close to but different from the $\mathbf{T}_{S}^{\vee}$-familiality of [21]. It would be instructive to better understand potential links between the two. Finally, in unpublished work, Fiore and Saville have considered the skew-monoidal case as a technical, intermediate setting tool [16]. Our proof of Theorem 2.15 is inspired by their notes.

\subsection{Plan}

We start in $§ 2$ by recalling Fiore et al.'s standard framework for syntax with binding and then familiality, explaining our move to the skew-monoidal setting, and proving our initiality and familiality results. We then continue in $\$ 3$ with a reminder and a reformulation of standard applicative bisimulation, which then guides the design of our abstract framework in $\$ 4$, where we prove our initiality result for vertical algebras. Finally, in $§ 5$, after briefly recalling Howe's method, we present the abstract Howe theorem and its proof, and devote $\S 6$ to a conclusion and some perspectives.

\subsection{Notation}

The category of (contravariant) presheaves on a category $\mathbb{C}$ is denoted by $\widehat{\mathbb{C}}$, the Yoneda embedding by $\mathbf{y}$, and $[A, B]$ is shorthand for the hom-set, or hom-category depending on the context, of morphisms $A \rightarrow B$.

\section{Syntax: familiality and substitution}

In this section, we explain Fiore et al.'s approach to specifying syntax with variable binding, on the particular case of pure $\lambda$-calculus. We then show that the obtained monad is not familial, hence move to a non-standard base category. This requires us to prove our generalised initiality result, together with familiality of the obtained monad.

\subsection{The standard setting}

The first step is to recall Fiore et al.'s standard theory. The relevant category for this, say $\mathscr{C}_{0}$, is the functor category

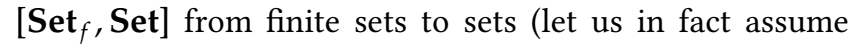
that Set $_{f}$ is a small category equivalent to finite sets, e.g., finite ordinals with arbitrary maps between them), or in other words the presheaf category $\widehat{\mathbb{C}_{0}}$, where $\mathbb{C}_{0}=\operatorname{Set}_{f}^{o p}$. An object $X$ is thus in particular a Set $_{f}$-indexed family of sets, and we think of $X(m)$ as the set of states with support $m$. If $X$ consists of terms, then $X(m)$ is typically the set of terms with free variables in $m$. The action of $X$ on morphisms $f: m \rightarrow n$ accounts for variable renaming: we think of $X(f): X(m) \rightarrow X(n)$ as renaming the free variables of terms in $X(m)$ according to $f$.

The basic ingredient for presenting our monad is the 'substitution' monoidal structure on $\mathscr{C}_{0}$ : the unit $I$ is the presheaf of variables, defined by $I(m)=m$, and elements of $(X \otimes$ $Y)(m)$ are pairs of some $x \in X(n)$ and a substitution $\sigma: n \rightarrow$ $Y(m)$, modulo the relation

$$
\left(\sigma, f \cdot x^{\prime}\right) \sim\left(\sigma \circ f, x^{\prime}\right),
$$

for any map $f: n^{\prime} \rightarrow n$ and $x^{\prime} \in X\left(n^{\prime}\right)$, where $f \cdot x^{\prime}$ denotes $X(f)\left(x^{\prime}\right)$. We denote by $x(\sigma)$ the equivalence class of $(\sigma, x)$.

A monoid is then an object $X \in \mathscr{C}_{0}$ equipped with morphisms $e: I \rightarrow X$ and $m: X \otimes X \rightarrow X$, satisfying standard associativity and unitality axioms.

Fiore et al.'s theory then tells us that $\lambda$-calculus syntax is the free $\Sigma_{0}^{\Lambda}$-monoid, i.e., the free monoid equipped with a compatible algebra structure for the pointed strong endofunctor

$$
\Sigma_{0}^{\Lambda}(X)(m)=X(m)^{2}+X(m+1) .
$$

An algebra for this endofunctor $\Sigma_{0}^{\Lambda}: \mathscr{C}_{0} \rightarrow \mathscr{C}_{0}$ is a presheaf $X$ equipped with application and $\lambda$-abstraction

$$
\left(-_{1}-{ }_{2}\right)_{m}: X(m)^{2} \rightarrow X(m) \text { and } \lambda_{m}: X(m+1) \rightarrow X(m),
$$

and the pointed strength specifies how standard, captureavoiding substitution should commute with both operations. Indeed, a pointed strength is a natural transformation

$$
\Sigma_{0}^{\Lambda}(X) \otimes Y \rightarrow \Sigma_{0}^{\Lambda}(X \otimes Y),
$$

where $Y$ ranges over pointed objects, i.e., objects in the coslice $I / \mathscr{C}$ under the monoidal unit $I$.

Example 2.1. The component of the pointed strength of $\Sigma_{0}^{\Lambda}$ at $X$ and $Y$ maps any pair $i n_{r}(e)(\sigma) \in\left(\Sigma_{0}^{\Lambda}(X) \otimes Y\right)(m)$ with $e \in X(n+1)$ (so $\left.i n_{r}(e) \in \Sigma_{0}^{\Lambda}(X)(n)\right)$ and $\sigma: n \rightarrow Y(m)$, to $i_{r}\left(e\left(\sigma^{\uparrow}\right)\right)$, where $\sigma^{\uparrow}$ denotes the composite

$$
n+1 \stackrel{\sigma+i n_{r}}{\longrightarrow} Y(m)+(m+1) \stackrel{\left[Y\left(i n_{l}\right), e_{m+1}\right]}{\longrightarrow} Y(m+1)
$$

where $e: I \rightarrow Y$ is the point of $Y$ - recalling that $I(m+1)=$ $m+1$ by definition. So, the pointed strength specifies that the given renaming $\sigma$ commutes with $\lambda$-abstraction, preserving the fresh variable.

By work of Fiore [17], the forgetful functor $\Sigma_{0}^{\Lambda}$-mon $\rightarrow$ $\mathscr{C}_{0}$ from $\Sigma_{0}^{\Lambda}$-monoids is monadic, and the induced monad $\mathscr{T}_{0}^{\Lambda}$ on $\mathscr{C}_{0}$ involves both operations, plus explicit substitution. It may be presented as a term language in which all explicit substitutions are pushed down towards the leaves, as in the grammar

$$
\mathscr{T}_{0}^{\Lambda}(X)(n) \ni e:=i\left|e_{1} e_{2}\right| \lambda \cdot e^{\prime} \mid o\left(e_{1}, \ldots, e_{m}\right),
$$

where $i \in n, o \in X(m)$ for some $m, e_{1}, e_{2}, \ldots, e_{m} \in \mathscr{T}_{0}^{\Lambda}(X)(n)$, and $e^{\prime} \in \mathscr{T}_{0}^{\Lambda}(X)(n+1)$.

Terminology 2.2. There are injections $n \hookrightarrow \mathscr{T}_{0}^{\Lambda}(X)(n)$ and $X(n) \hookrightarrow \mathscr{T}_{0}^{\Lambda}(X)(n)$, which are both close in spirit to injections of variables into terms. In order to distinguish them, we think of the former as an injection of variables, and of the latter as an injection of constants. 


\subsection{Failure of familiality}

As announced in the introduction, the characterisation of syntax with variable binding through $\Sigma_{0}$-monoids is problematic for us because the obtained monad $\mathscr{T}_{0}^{\Lambda}$ is not familial, as we now explain. Let us first start by briefly recalling familiality $[9,12,40,41]$. One way to understand it is as providing an abstract counterpart to multi-hole contexts, or linear terms, in the following sense.

Example 2.3. Consider the 'free monoid' $\operatorname{monad} M$ on Set, which maps any set $X$ to the set $\sum_{n} X^{n}$ of finite sequences of elements. Any sequence in $M(X)$, say $\left(x_{1}, \ldots, x_{n}\right)$, viewed as a map $1 \rightarrow M(X)$, decomposes as the corresponding linear sequence $(1, \ldots, n)$ over $\{1, \ldots, n\}$, and the renaming mapping any $i$ to $x_{i}$. Equivalently, using $n$ as shorthand for $\{1, \ldots, n\}$, it factors as $1 \stackrel{(1, \ldots, n)}{\longrightarrow} M(n) \stackrel{M\left[x_{i}\right]_{i}}{\longrightarrow} M(X)$. In fact, linear sequences enjoy the following 'genericness' property.

Definition 2.4. Given any functor $F: \mathscr{A} \rightarrow \mathscr{B}$, a morphism $\xi: B \rightarrow F(A)$ is $F$-generic (or generic for short) whenever any square of the form below (solid) admits a unique lifting $k$ (dashed) such that $F(k) \circ \xi=\chi$ and $g \circ k=f$.

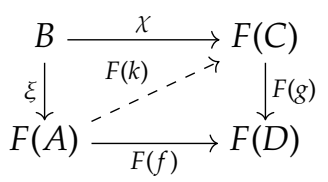

$F$ is familial iff any morphism $f: B \rightarrow F(X)$ admits a generic factorisation, i.e., factors as $B \stackrel{\xi}{\rightarrow} F(A) \stackrel{F(h)}{\longrightarrow} F(X)$ with $\xi$ generic. Any morphism of the form $F(h)$ is deemed free.

We have the following important alternative characterisations in the case of presheaf categories, recalling from Paré [30] that a functor preserves connected limits iff it preserves wide pullbacks.

Theorem 2.5 (Weber [40, Theorem 8.1]). For any accessible endofunctor $F$ on any presheaf category $\widehat{\mathbb{C}}$, the following are equivalent:

(i) F is familial;

(ii) F preserves wide pullbacks;

(iii) there is a functor $E: \operatorname{el}(F(1)) \rightarrow \widehat{\mathbb{C}}$ such that

$$
F(X)(c) \cong \sum_{x \in F(1)(c)} \widehat{\mathbb{C}}(E(c, x), X),
$$

naturally in $X$ and $c$.

Recall from MacLane and Moerdijk [29] that the category of elements $\mathrm{el}(X)$ of a presheaf $X$ over any category $\mathbb{C}$ has pairs $(c, x)$ with $x \in X(c)$ as objects, and as morphisms $(c, x) \rightarrow\left(c^{\prime}, x^{\prime}\right)$ all morphisms $f: c \rightarrow c^{\prime}$ such that $X(f)\left(x^{\prime}\right)=$ $x$.

Proposition 2.6. The functor $\Sigma_{0}^{\Lambda}$ is familial, but the monad $\mathscr{T}_{0}^{\Lambda}$ is not.
Proof sketch. Familiality of $\Sigma_{0}^{\Lambda}$ is easy by the theorem, since we have $\Sigma_{0}^{\Lambda}(X)(n) \cong\left[\mathbf{y}_{n}+\mathbf{y}_{n}, X\right]+\left[\mathbf{y}_{n+1}, X\right]$.

For non-familiality of $\mathscr{T}_{0}^{\Lambda}$, the proof is not particularly illuminating, but here is a hopefully helpful intuitive argument.

For any closed term $e$, viewed by action of $0 \rightarrow \mathbf{y}_{1}$ as an element of $\mathscr{T}_{0}^{\Lambda}\left(\mathbf{y}_{1}\right)(0)$, a natural candidate generic is the term $i d_{1}(e)$, viewed by Yoneda as a morphism $\mathbf{y}_{0} \rightarrow \mathscr{T}_{0}^{\Lambda}\left(\mathbf{y}_{1}\right)$, where we think of $i d_{1}$ as a unary constant, to which we feed $e$ as argument by explicit substitution.

Let us show that it is in fact not generic. Indeed, under the action of $\mathbf{y}_{!}: \mathbf{y}_{1} \rightarrow \mathbf{y}_{0}$, id $d_{1}$ is mapped to !: $0 \rightarrow 1$ viewed as an element of $\mathbf{y}_{0}(1)=\operatorname{Set}(0,1)$, so by (1) $i d_{1}(e)$ is mapped by $\mathscr{T}_{0}^{\Lambda}\left(\mathbf{y}_{!}\right)$to

$$
!(e)=i d_{0}(e \circ !)=i d_{0}() \text {. }
$$

Of course, this would hold for any closed $e^{\prime} \neq e$, so that we get a commuting square

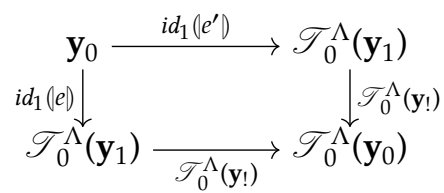

with no filler. In fact, because there are infinitely many distinct closed terms, we get infinitely many factorisations of the diagonal $i d_{0}()$, for which no candidate generic can provide enough fillers.

\subsection{Familial syntax}

So the standard monad for $\lambda$-calculus syntax is not familial, which prevents us from applying the methods of [21]. From the proof of Proposition 2.6, clearly, the problem comes from quotienting by (1), so we move to the more rigid category $\mathscr{C}_{0}=[\mathbb{N}$, Set $]$ of $\mathbb{N}$-indexed families. A first difficulty is that it does not fit Fiore et al.'s general framework. Indeed, the natural tensor product on $[\mathbb{N}$, Set], defined by

$$
(A \otimes B)(n)=\sum_{m} A(m) \times B(n)^{m},
$$

is only associative and unital up to non-invertible arrows, which makes $\mathscr{C}_{0}$ a skew-monoidal category.

Notation 2.7. An element of $(A \otimes B)(n)$ is a triple $(m, a, \beta)$ with $a \in A(m)$ and $\beta: m \rightarrow B(n)$. Leaving $m$ implicit and thinking of the triple as an explicit substitution, we denote it again by $a(\beta)$.

Example 2.8. Associativity fails because elements of $(A \otimes$ $B) \otimes C$ have the form $\left(a\left(b_{1}, \ldots, b_{m}\right)\right)(\sigma)$, while those of $A \otimes$ $(B \otimes C)$ have the form $a\left(b_{1}\left(\sigma_{1}\right), \ldots, b_{m}\left(\sigma_{m}\right)\right)$ : we can map the former to the latter by pushing $\sigma$ into the substitution $\left(b_{1}, \ldots, b_{m}\right)$, but not conversely, because $\sigma_{1}, \ldots$, and $\sigma_{m}$ may not all be the same. In [Set $f$, Set],$a\left(b_{1}\left(\sigma_{1}\right), \ldots, b_{m}\left(\sigma_{m}\right)\right)$ may be given the desired form thanks to (1), by forming the compound substitution $\left[\sigma_{1}, \ldots, \sigma_{m}\right]: p_{1}+\ldots+p_{m} \rightarrow C(n)$. 
So we should generalise Fiore et al.'s theory to skew-monoidal categories. But in fact, moving to the category $\mathscr{C}_{0}$ generates a second difficulty, namely that pointed strong endofunctors become inadequate. Indeed, e.g., the pointed strength $\Sigma_{0}^{\Lambda}(X) \otimes Y \rightarrow \Sigma_{0}^{\Lambda}(X \otimes Y)$ of $\Sigma_{0}^{\Lambda}$ on [Set , $_{f}$, Set] relies both on variables and renaming in $Y$ (as shown by the presence of $e_{m+1}$ and $Y\left(i n_{l}\right)$ in the definition (3) of $\left.\sigma^{\uparrow}\right)$. So the notion of strength we need for endofunctors on $\mathscr{C}_{0}$ should assume that $Y$ comes equipped with variables and renaming. Variables are given by a point as before, while renaming is taken care of by I-module structure, in the following sense.

Definition 2.9. In any skew-monoidal category $\mathscr{C}$, for any monoid $X$, the category $X$-mod of (right) $X$-modules has as objects all $M \in \mathscr{C}$ equipped with an action $r: M \otimes X \rightarrow$ $M$, satisfying two standard coherence conditions. A module morphism is a morphism commuting with action.

As desired, an action $Y \otimes I \rightarrow Y$ yields for all $n$ a map $(Y \otimes I)(n)=\sum_{m} Y(m) \times n^{m} \rightarrow Y(n)$ giving the action of morphisms $m \rightarrow n$ on $Y(m)$. The unit $I$ is canonically an $I-$ module, and a point for an $I$-module $Y$ is a morphism $I \rightarrow$ $Y$ in $I$-mod. Thus, the appropriate category for $Y$ is the following coslice category.

Definition 2.10. Let the category $I-\bmod _{I}$ of pointed $I-$ mo- $^{-}$ dules be the coslice $I / I$-mod.

Let us now define the appropriate notion of strength, similarly to [25] (generalising [17, I.1.2]). We first equip I - $\bmod _{I}$ with skew-monoidal structure. Following [24, (8.1)], tensor product of (pointed) $I$-modules is given by the following coequaliser in $\mathscr{C}$, where $r_{X}: X \otimes I \rightarrow X$ is the $I$-module structure on $X$.

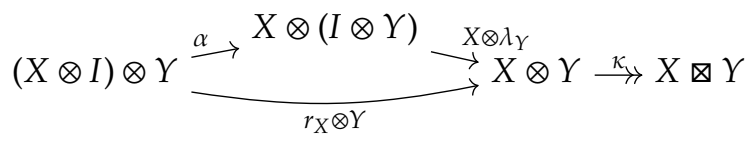

By [24, Theorem 8.1], I- $\mathbf{m o d}$ is a skew-monoidal category (with invertible right unit), and the forgetful functor is monoidal and creates monoids. In fact, this extends to $I-\mathbf{m o d}_{I}$, and we define:

Definition 2.11. A structural strength on a functor $F: \mathscr{C}^{n} \rightarrow$ $\mathscr{C}$ is a natural transformation st with components

$$
s t_{A, Y}: F(A) \otimes Y \rightarrow F(A \otimes Y)
$$

where $Y \in I-\bmod _{I}, A=\left(X_{1}, \ldots, X_{n}\right)$, and $A \otimes Y:=\left(X_{1} \otimes\right.$ $\left.Y, \ldots, X_{n} \otimes Y\right)$, making the following diagrams commute.

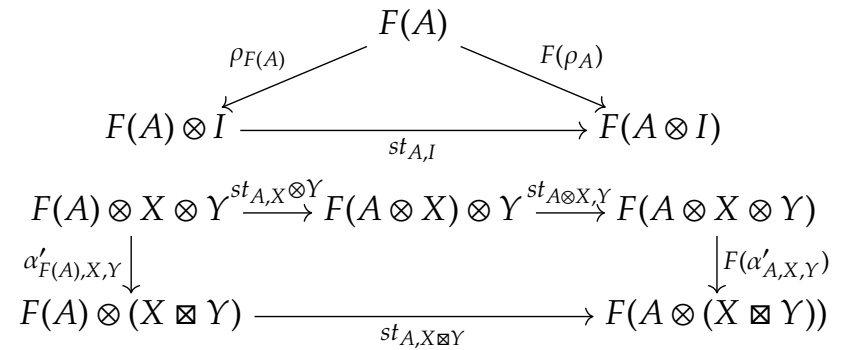

where $\alpha_{A, B, C}^{\prime}$ is $(A \otimes \kappa) \circ \alpha_{A, B, C}$.

Example 2.12. The endofunctor $\Sigma_{0}^{\Lambda}: \widehat{\mathbb{N}} \rightarrow \widehat{\mathbb{N}}$ for the syntax of pure $\lambda$-calculus is defined by the same formula (2) as before. Let us now construct its structural strength. For any pointed $I$-module $(Y, e: I \rightarrow Y, r: Y \otimes I \rightarrow Y)$ and map $f: m \rightarrow n$, the map $\bar{r}_{n}: \sum_{m} Y(m) \times n^{m} \rightarrow Y(n)$ specialises to $Y(f):=\bar{r}_{n}(-, f): Y(m) \rightarrow Y(n)$. We may thus define the desired structural strength by

$$
\begin{aligned}
s t_{X, Y, n}: \quad\left(\Sigma_{0}^{\Lambda}(X) \otimes Y\right)(n) & \rightarrow \Sigma_{0}^{\Lambda}(X \otimes Y)(n) \\
\left(\operatorname{in}_{l}(y, z)\right)(v) & \mapsto i n_{l}(y(v), z(v)) \\
\left(\operatorname{in}_{r}(x)\right)(v) & \mapsto i n_{r}\left(x\left(v^{\uparrow}\right)\right)
\end{aligned}
$$

where we assume $v: m \rightarrow Y(n), x \in X(m+1)$, and $v^{\uparrow}: m+$ $1 \rightarrow Y(n+1)$ is as in (3).

Observing that any monoid is in particular a pointed $I$ module, we may define $F$-monoids in the new setting.

Definition 2.13. For any structurally strong endofunctor $F$, an $F$-monoid is an object $X$ equipped with $F$-algebra and monoid structures $a: F(X) \rightarrow X$ and $I \stackrel{e}{\rightarrow} X \stackrel{m}{\leftarrow} X \otimes X$, such that the following diagram commutes.

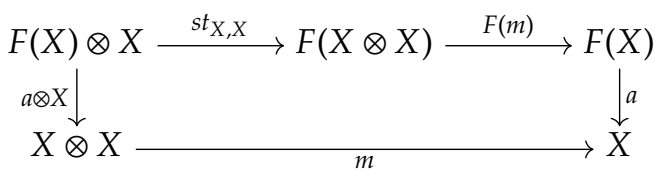

Proposition 2.14. For any structurally strong endofunctor F, F-monoids form a category F-mon, whose morphisms are morphisms of underlying objects that respect both the monoid and algebra structure.

Our first main result is:

Theorem 2.15. For any finitary, structurally strong endofunctor $F$ on a cocomplete skew-monoidal category $\mathscr{C}$, if the tensor preserves colimits on the left and directed colimits on the right, then the forgetful functor $\mathscr{U}^{F}: F-$ mon $\rightarrow \mathscr{C}$ is monadic, and the free $F$-monoid on any $X \in \mathscr{C}$ has carrier $\mu A .(I+F(A)+$ $X \otimes A)$, i.e., the colimit of the chain

$$
F_{X}^{0}(0) \stackrel{\partial_{X}^{0}}{\longrightarrow} F_{X}^{1}(0) \rightarrow \ldots \rightarrow F_{X}^{n}(0) \stackrel{\partial_{X}^{n}}{\longrightarrow} F_{X}^{n+1}(0) \rightarrow \ldots,
$$

where

- $F_{X}: \mathscr{C} \rightarrow \mathscr{C}$ maps any $A$ to $I+F(A)+X \otimes A$;

- $L^{0}=I d$ and $L^{n+1}=L \circ L^{n}$, for any endofunctor $L$ on any category; 
- $\partial_{X}^{0}: 0=F_{X}^{0}(0) \rightarrow F_{X}^{1}(0)$ is the unique such map;

- $\partial_{X}^{n+1}: F_{X}\left(F_{X}^{n}(0)\right) \rightarrow F_{X}\left(F_{X}^{n+1}(0)\right)$ denotes $F_{X}\left(\partial_{X}^{n}\right)$.

Notation 2.16. Let $F^{\circledast}$ denote the 'free $F$-monoid' monad. We sometimes abbreviate $F^{\circledast}(X)$ as $X^{\circledast}$ when $F$ is clear.

Let us now prove that $F^{\circledast}$ is familial as desired, and furthermore that the unit $\eta_{F}: F \rightarrow F^{\circledast}$ preserves familiality, in the sense of preserving generics. In order to establish this, we rely on:

Proposition 2.17 ([40, Proposition 5.10(2)]). Any cartesian natural transformation, i.e., one whose naturality squares are pullbacks, preserves generic morphisms.

So we want to show that $\eta_{F}$ is cartesian. This will rely on properties of $\mathscr{C}$, that we first show are satisfied by $\widehat{\mathbb{N}}$.

Example 2.18. As a presheaf category, $\widehat{\mathbb{N}}$ is of course $e x$ tensive [10]. Furthermore, each $\rho_{X}: X \rightarrow X \otimes I$ is obviously monic, and $\rho$ will be cartesian if each square of the form

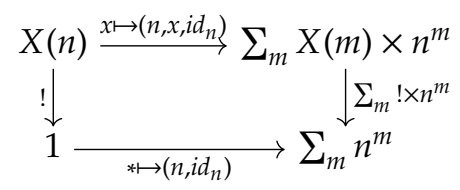

is a pullback. But if $\left(\sum_{m} ! \times n^{m}\right)(x(f))=\left(n, i d_{n}\right)$ for some $x(f)$, then $(m, f)=\left(n, i d_{n}\right)$, so $x$ is the desired unique element of $X(n)$ with specified projections.

Tensor product moreover preserves wide pullbacks, hence is familial, on both sides. On the left, this holds because coproducts commute with connected limits in presheaf categories in general, hence wide pullbacks. On the right, it holds for the same reason, plus the fact that each functor $X \mapsto X^{n}$ in Set also preserves wide pullbacks.

Abstracting over this situation, we obtain:

Theorem 2.19. In the situation of Theorem 2.15, if

- $\mathscr{C}$ is extensive,

- $\rho$ is cartesian and monic, and

- $\otimes$ and $F$ are familial,

then $F^{\circledast}$ is familial and the natural transformation $\eta_{F}: F \rightarrow$ $F^{\circledast}$ is monic and cartesian.

\section{Evaluation and applicative bisimulation}

In the previous section, we have amended Fiore et al.'s standard framework to make it compatible with the familial approach to operational semantics. The next step is to deal with transitions. For this, we start in this section by analysing standard, syntactic applicative bisimulation. Guided by our findings, we will design our abstract setting in the next section.

\subsection{Substitution-closed bisimulation}

Standardly, evaluation is inductively defined by the rules

$$
\overline{\lambda x . e \Downarrow \lambda x . e} \quad \frac{e_{1} \Downarrow \lambda x \cdot e_{1}^{\prime} \quad e_{1}^{\prime}\left[x \mapsto e_{2}\right] \Downarrow e_{3}}{e_{1} e_{2} \Downarrow e_{3}}
$$

and applicative bisimilarity is introduced in two stages. First, one defines applicative bisimulation on closed terms.

Definition 3.1. A relation $R$ over closed $\lambda$-terms is an applicative bisimulation iff $e_{1} R e_{2}$ and $e_{1} \Downarrow \lambda x$. $e_{1}^{\prime}$ entails the existence of $e_{2}^{\prime}$ such that $e_{2} \Downarrow \lambda x . e_{2}^{\prime}$ and, for all terms $e$, $e_{1}^{\prime}[x \mapsto e] R e_{2}^{\prime}[x \mapsto e]$, and symmetrically.

Applicative bisimulations are closed under unions, and so there is a largest applicative bisimulation called applicative bisimilarity and denoted by $\sim$. Then comes the second stage:

Definition 3.2. The open extension of a relation $R$ on closed terms is the relation $R^{\circ}$ on potentially open terms such that $e R^{\circ} e^{\prime}$ iff for all closed substitutions $\sigma$ covering all involved free variables we have $e[\sigma] R e^{\prime}[\sigma]$.

Lemma 3.3. The open extension of any relation $R$ is equivalently the greatest relation $R^{\prime}$ on potentially open terms such that ife $R^{\prime} e^{\prime}$, then for all closed substitutions $\sigma$ covering all involved free variables we have $e[\sigma] R e^{\prime}[\sigma]$.

Proof. By definition, $R \square$ satisfies the condition. To see that it is the greatest such relation, consider any $R^{\prime}$ satisfying it: for all $e R^{\prime} e^{\prime}$, we have $e[\sigma] R e^{\prime}[\sigma]$ for all closing $\sigma$, hence $e R \square e^{\prime}$ by definition; thus $R^{\prime} \subseteq R \square$ as desired.

The result we wish to prove in the abstract setting is:

Theorem 3.4 (See [31] for a historical account). The open extension $\sim^{\circ}$ of applicative bisimilarity is a congruence, i.e., it is an equivalence relation, and furthermore

- $e_{1} \sim^{\circ} e_{2}$ entails $\lambda x . e_{1} \sim^{\circ} \lambda x . e_{2}$ for all $x$;

- $e_{1} \sim^{\circ} e_{2}$ and $e_{1}^{\prime} \sim^{\circ} e_{2}^{\prime}$ entail $e_{1} e_{1}^{\prime} \sim^{\circ} e_{2} e_{2}^{\prime}$.

We take a slightly different viewpoint here, starting from the following observation.

Lemma 3.5. The open extension of applicative bisimilarity is equivalently the greatest substitution-closed opening bisimulation, i.e., the greatest relation $R$ on potentially open terms such that

- $e_{1} R e_{2}$ entails $e_{1}[x \mapsto e] R e_{2}[x \mapsto e]$ for anye;

- if $e_{1}$ and $e_{2}$ are closed, $e_{1} R e_{2}$, and $e_{1} \rightarrow^{\star} \lambda x . e_{1}^{\prime}$ then $e_{2} \rightarrow^{\star} \lambda x . e_{2}^{\prime}$ with $e_{1}^{\prime} R e_{2}^{\prime}$, and symmetrically.

Proof. The relation $\sim^{\circ}$ is straightforwardly a substitutionclosed opening bisimulation. But any substitution-closed opening bisimulation $R$ restricts to an applicative bisimulation on closed terms, which is thus included in $\sim$. On open terms, if $e_{1} R e_{2}$, then for all closing substitutions $\sigma$ we have by substitution-closure of $R$ that $e_{1}[\sigma] R e_{2}[\sigma]$, hence $e_{1}[\sigma] \sim e_{2}[\sigma]$ and so $e_{1} \sim e_{2}$ by definition. 
When designing our abstract framework, we should thus be able to model two things: opening bisimulation and substitution-closed relations.

The latter is easy, remembering that tensor product on $\widehat{\mathbb{N}}$ is a sort of explicit substitution. Indeed, substitution-closed relations on any object $X$ are relations in the category of $X$-modules (in the sense of Definition 2.9), i.e., objects $R$ equipped with a map $R \otimes X \rightarrow R$ over $X^{2}$.

For modelling opening bisimulation, we should extend $\widehat{\mathbb{N}}$, which is only good at modelling the syntax of $\lambda$-calculus: in order to also model transitions, let us consider the following category.

Definition 3.6. Let $\mathbb{C}$ denote the free category on the graph with a vertex $n$ for all $n \in \mathbb{N}$, plus a vertex $\Downarrow$, with only edges $0 \stackrel{s}{\rightarrow} \Downarrow \stackrel{t}{\leftarrow} 1$. Let $\mathscr{C}=\widehat{\mathbb{C}}$.

Objects $X \in \widehat{\mathbb{C}}$ are $\mathbb{N}$-indexed families, together with a set $X(\Downarrow)$ of transitions, the source and target of any $r \in X(\Downarrow)$ are given by $X(s): X(\Downarrow) \rightarrow X(0)$ and $X(t): X(\Downarrow) \rightarrow X(1)$.

The full embedding $\mathbf{i}: \mathbb{N} \hookrightarrow \mathbb{C}$ induces by restriction and left Kan extension a coreflection

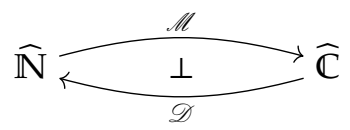

(where $\mathscr{M}$ stands for 'monter', get up in French, and $\mathscr{D}$ for 'descendre', get down).

Because targets of transitions in any $X \in \widehat{\mathbb{C}}$ are in $X(1)$, we may define the transition system for big-step $\lambda$-calculus so as to make opening bisimulation the natural notion of bisimulation: we take it to be the presheaf $X$ on $\mathbb{C}$, with $X(\Downarrow)$ the set of evaluation proofs $r: e \Downarrow \lambda x \cdot e^{\prime}$, in the standard sense, $X(s)(r)=e \in X(0)$, and $X(t)(r)=e^{\prime} \in X(1)$. This way, any term evaluates to the body of its value, which, combined with substitution-closedness, achieves the desired effect. We thus consider the modified evaluation rules

$$
\frac{e_{1} \Downarrow e_{1}^{\prime} \quad e_{1}^{\prime}\left[e_{2}\right] \Downarrow e_{3}}{e_{1} e_{2} \Downarrow e_{3}} \quad \overline{\lambda . e \Downarrow e}
$$

where $e_{1}^{\prime}, e_{3} \in X(1)$ and $e_{1}^{\prime}\left[e_{2}\right]$ denotes standard captureavoiding substitution of the unique free variable of $e_{1}^{\prime}$.

Following the open maps approach to bisimulation [21, 23], functional opening bisimulations may be defined as arrows $f: R \rightarrow X$ such that for all commuting squares as the solid part of

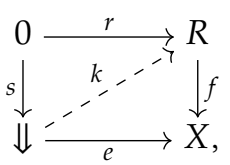

there is a filling $k$ as shown (dashed), making both triangles commute. Opening bisimulations are then relations $R \hookrightarrow$ $X^{2}$ whose projections are functional opening bisimulations.
Proposition 3.7. Substitution-closed bisimulations, or $X-$ bisimulations, i.e., bisimulations of X-modules, are closed under unions and hence admit a greatest element, called X-bisimilarity, or substitution-closed bisimilarity.

With this definition, we have:

Proposition 3.8. Substitution-closed bisimilarity is precisely the open extension of applicative bisimilarity.

\subsection{Vertical algebras}

Naively, following [21], the next step should be to describe the syntax and evaluation rules of big-step $\lambda$-calculus as the initial algebra for some monad $\mathscr{T}$ on $\widehat{\mathbb{C}}$. The idea is that $\mathscr{T}(A)(n)$ should consist of terms with $n$ free variables and constants in all $A(m)$ 's, while $\mathscr{T}(A)(\Downarrow)$ should consist of transition proofs with constants in $A(\Downarrow)$ (remembering Terminology 2.2). As explained in $\S 1$, the problem lies in the $\beta$-rule (modified or not), whose premises use substitution. In order for the monad $\mathscr{T}$ to make sense, this requires the argument $A$ to feature some notion of term substitution. In other situations, one could even imagine requiring $A$ to be a model of the syntax. We will thus define $\mathscr{T}$ as a monad on the pullback category

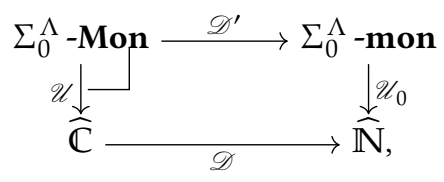

whose objects we call transition $\Sigma_{0}^{\Lambda}$-monoids, are presheaves $A$ on $\mathbb{C}$, equipped with $\Sigma_{0}^{\Lambda}$-monoid structure on the underlying presheaf $\mathscr{D}(A)$ on $\mathbb{N}$.

Let us define $\mathscr{T}$ through a generating endofunctor: given any $\Sigma_{0}^{\Lambda}$-monoid $A \in \widehat{\mathbb{C}}$, we let

$$
\begin{aligned}
& \Sigma_{1}^{\Lambda}(A)(n)=\left(\Sigma_{0}^{\Lambda}\right)^{\circledast}(\mathscr{D}(A))(n) \\
& \Sigma_{1}^{\Lambda}(A)(\Downarrow)=A(1)+\operatorname{ar}^{\beta}(A),
\end{aligned}
$$

for all $n \in \mathbb{N}$, where

- $\left(\Sigma_{0}^{\Lambda}\right)^{\circledast}$ is as in Notation 2.16 ,

- $A(1)$ accounts for Rule (5, right), and

- $\operatorname{ar}^{\beta}(A)$ accounts for Rule (5, left): it denotes the set of triples $\left(r_{1}, e_{2}, r_{2}\right) \in A(\Downarrow) \times A(0) \times A(\Downarrow)$, such that $r_{2} \cdot s=$ $\left(r_{1} \cdot t\right)\left[e_{2}\right]$ (where '. ' is as in (1) but for contravariant presheaves, e.g., $\left.r_{2} \cdot s=A(s)\left(r_{2}\right)\right)$.

The source and target maps are defined as expected. E.g., any $\left(r_{1}, e_{2}, r_{2}\right)$ with $r_{1}: e_{1} \Downarrow e_{1}^{\prime}$ and $r_{2}: e_{1}^{\prime}\left[e_{2}\right] \Downarrow e_{3}$ has source and target $e_{1}(\mid) e_{2}(l)$ and $e_{3}(1)$, respectively.

Remark 3.9. Substitution $e_{1}^{\prime}\left[e_{2}\right]$ follows from the monoid structure of $A$. We should also prove that $\Sigma_{1}^{\Lambda}(A)$ is a transition $\Sigma_{0}^{\Lambda}$-monoid, which holds because $\left(\Sigma_{0}^{\Lambda}\right)^{\circledast}(\mathscr{D}(A))$ is a $\Sigma_{0}^{\Lambda}$-monoid by construction. In fact, we have $\mathscr{D}^{\prime}\left(\Sigma_{1}^{\Lambda}(A)\right)=$ $\mathscr{K}_{0}\left(\mathscr{D}^{\prime}(A)\right)$, where $\mathscr{K}_{0}$ is the comonad induced by the adjunction $\mathscr{L}_{0} \dashv \mathscr{U}_{0}$. 
We have thus organised the operational rules into an endofunctor on transition $\Sigma_{0}^{\Lambda}$-monoids. However, we do not yet have any monad, or, worse, any notion of model. Indeed, any $\Sigma_{1}^{\Lambda}$-algebra $A$ has two underlying algebra structures for the endofunctor $\left(\Sigma_{0}^{\Lambda}\right)^{\circledast}$ : one from the $\Sigma_{0}^{\Lambda}$-algebra structure of $\mathscr{D}^{\prime}(A)$, and the other from the fact that $\Sigma_{1}^{\Lambda}$ coincides with $\left(\Sigma_{0}^{\Lambda}\right)^{\circledast}$ on the syntactic level.

But in fact, we may construct the following modified variant $\check{\Sigma}_{1}^{\Lambda}$ of $\Sigma_{1}^{\Lambda}$ by setting

$$
\check{\Sigma}_{1}^{\Lambda}(A)(n)=A(n) \quad \text { and } \quad \check{\Sigma}_{1}^{\Lambda}(A)(\Downarrow)=\Sigma_{1}^{\Lambda}(A)(\Downarrow),
$$

with source and target maps given by composition with the $\left(\Sigma_{0}^{\Lambda}\right)^{\circledast}$-algebra structure of $A$. The relevant models are just $\check{\Sigma}_{1}^{\Lambda}$-algebras whose structure map is sent to the identity by the forgetful functor $\Sigma_{0}^{\Lambda}$-Mon $\rightarrow \Sigma_{0}^{\Lambda}$-mon. Following Ahrens et al. [4, 20], we deem such algebras vertical.

As we will prove below (Theorem 4.20), free vertical algebras may be characterised inductively, which in the present case means that the free vertical algebra over any transition $\Sigma_{0}^{\Lambda}$-monoid is inductively defined by the modified rules (5), augmented with an axiom

$$
\frac{o \Downarrow o^{\prime} \text { in } A}{o\left(b \Downarrow o^{\prime}(1)\right.}
$$

Lifting the adjunction of Theorem 2.15 from $\widehat{\mathbb{C}_{0}}$ to $\widehat{\mathbb{C}}$, we obtain a chain of monadic adjunctions

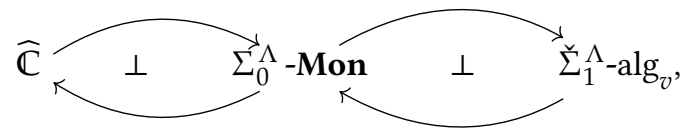

where $\check{\Sigma}_{1}^{\Lambda}-\operatorname{alg}_{v}$ is the category of vertical $\check{\Sigma}_{1}^{\Lambda}$-algebras.

We at last arrive at a setting in which to study the question we started with, in its new guise: is substitution-closed bisimilarity a congruence in the initial vertical algebra?

However, there is a last issue that prevents us from directly applying the techniques of [21]: calling $\mathscr{T}^{\Lambda}$ the obtained monad over $\mathscr{C}=\widehat{\mathbb{C}}$, compositionality fails, i.e., the multiplication $\mu$ for $\mathscr{T}^{\Lambda}$ is not a functional bisimulation.

Example 3.10. The problem is essentially as follows. Monad multiplication takes a term of terms (i.e., constants are terms), and performs the substitution: typically, for any such terms of terms $E_{1}, \ldots, E_{n}$, we have

$$
\mu\left(e\left(E_{1}, \ldots, E_{m}\right)\right)=e\left[\mu\left(E_{1}\right), \ldots, \mu\left(E_{m}\right)\right],
$$

i.e., $e$ where each variable $j \in m$ is replaced with $\mu\left(E_{j}\right)$. E.g., over $m=2=\{x, y\}$, the term of terms $(x y)(\lambda z . z, \lambda z . z)$ is mapped to the closed redex $e:=(\lambda z . z)(\lambda z . z)$. Since we have $e \Downarrow z$, compositionality would require the existence of a transition $(x y)(\lambda z . z, \lambda z . z) \Downarrow E^{\prime}$, for some $E^{\prime}$. However, the only transition rule whose conclusion has as source a term of the form $o(\ldots)$ is $(10)$, in which $o$ is nullary, so it cannot apply here.
Although we cannot hope free vertical algebras to be compositional, we in fact only need a restricted form of compositionality, demanding that for any commuting square as the solid part below,

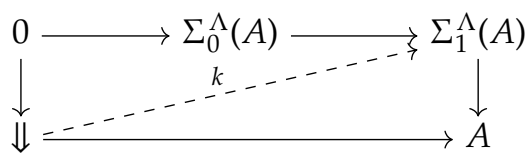

there exists a lifting $k$ as shown. In this case, we call $A$ a weakly compositional algebra.

Remark 3.11. The endofunctor $\Sigma_{0}^{\Lambda}$ really acts on $\mathscr{C}_{0}$, not $\mathscr{C}$, so some implicit casting is going on: the whole diagram takes place in $\mathscr{C}$, so $\Sigma_{1}^{\Lambda}(A)$ and $A$ should be considered as shorthand for their images through the forgetful functor $\mathscr{U}: \Sigma_{0}^{\Lambda}$-Mon $\rightarrow \mathscr{C}$, while $\Sigma_{0}^{\Lambda}(A)$ is in fact shorthand for $\mathscr{M}\left(\Sigma_{0}^{\Lambda}(\mathscr{D}(\mathscr{U}(A)))\right)$.

Intuitively, by Yoneda, weak compositionality says that for any transition $r \in A(\Downarrow)$ whose source is obtained by evaluating a term $e$ of depth $1, r$ is obtained by evaluating a transition term $R \in \Sigma_{1}^{\Lambda}(A)(\Downarrow)$, whose source is $e$, all as in

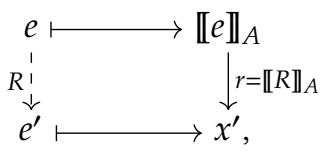

where $\llbracket-\rrbracket_{A}: \Sigma_{1}^{\Lambda}(A) \rightarrow A$ denotes the algebra structure. In other terms, $\llbracket-\mathbb{\rrbracket}_{A}$ has the functional bisimulation property restricted to terms of depth 1 without explicit substitution.

\section{An abstract setting for Howe's method}

We are now ready for abstracting over big-step $\lambda$-calculus.

\subsection{Howe contexts}

We start by axiomatising the ambient setting for transition systems, notably their layered nature. Let us recall that any fully faithful functor $F: \mathbb{C} \rightarrow \mathbb{D}$ induces a full coreflection

$$
\sum_{F}: \widehat{\mathbb{C}} \underset{\leftarrow}{\perp} \mathbb{D}: \Delta_{F},
$$

where $\Sigma_{F}$ and $\Delta_{F}$ respectively denote left Kan extension and restriction along $F^{o p}$. By the triangle identities, $\Delta_{F}\left(\varepsilon_{X}\right)$ and $\varepsilon_{\Sigma_{F}(C)}$ are isomorphisms for all $X \in \widehat{\mathbb{D}}$ and $C \in \widehat{\mathbb{C}}$.

Furthermore, let 2 denote the poset $\{0<1\}$ viewed as a category.

Definition 4.1. A Howe context consists of a small category $\mathbb{C}$ together with a functor $\mathbf{p}: \mathbb{C} \rightarrow 2$, equipped with skewmonoidal structure on the presheaf category $\widehat{\mathbb{C}_{0}}$, where $\mathbb{C}_{0}$ denotes the fibre of $\mathbf{p}$ over 0 , satisfying

(H1) each functor $-\otimes C$ is familial and preserves colimits,

(H2) each functor $C \otimes-$ is familial and preserves filtered colimits, and 
(H3) denoting by i: $\mathbb{C}_{0} \hookrightarrow \mathbb{C}$ the canonical embedding, the counit $\varepsilon_{L}: \sum_{\mathbf{i}} \Delta_{\mathbf{i}}(L) \rightarrow L$ is a copairing $[s, t]: P+$ $Q \rightarrow L$ with $P, Q \in \mathbb{C}_{0}$, for all $L \in \mathbb{C} \backslash \mathbb{C}_{0}$.

In particular, each $\Delta_{\mathbf{i}}[s, t]$ is an isomorphism, so any morphism from some $R \in \mathbb{C}_{0}$ to $L$ factors uniquely through either $s$ or $t$.

Notation 4.2. We respectively call objects of $\mathbb{C}, \mathbb{C}_{0}$, and $\mathbb{C} \backslash \mathbb{C}_{0}$ basic objects, state types, and transition types, and let $\mathscr{C}_{0}=\widehat{\mathbb{C}_{0}}, \mathscr{C}=\widehat{\mathbb{C}}, \mathscr{M}=\Sigma_{\mathrm{i}}$, and $\mathscr{D}=\Delta_{\mathrm{i}}$. We often omit $\mathscr{M}$ and $\mathscr{D}$ for readability.

Let us note that $\mathscr{M}$ extends a presheaf $X \in \widehat{\mathbb{C}_{0}}$ by mapping any transition type to the empty set.

Example 4.3. For pure $\lambda$-calculus, $\varepsilon_{\Downarrow}$ is the copairing $0+$ $1 \stackrel{[s, t]}{\longrightarrow} \Downarrow$ (or rather its image under the Yoneda embedding). We have already explained familiality in Example 2.18. Regarding colimits, for $-\otimes X$, the considered colimits should merely commute with coproducts, hence $-\otimes X$ commutes with all colimits. For $X \otimes-$, the considered colimits should commute with coproducts and finite products in sets, so the best we can say in general is that $X \otimes-$ commutes with sifted colimits, hence in particular with filtered colimits.

Let us conclude this section with two crucial properties.

Lemma 4.4. The functor $\mathscr{D}: \mathscr{C} \rightarrow \mathscr{C}_{0}$ is a bifibration, for which opcartesian liftings are isos at transitions types.

Proof. The opcartesian lifting $f \cdot X$ of any given $X \in \mathscr{C}$ along $f: \mathscr{D}(X) \rightarrow C$ is given by taking $(f \cdot X)(P)=C(P)$ for all $P \in \mathbb{C}_{0}$ and, $(f \cdot X)(L)=X(L)$ for all transition types $L \leftarrow P+Q:[s, t]$, with $(f \cdot X)(s)$ and $(f \cdot X)(t)$ given by composition, e.g., $X(L) \rightarrow X(P) \rightarrow C(P)$.

The cartesian lifting $X \cdot f$ of any $X \in \mathscr{C}$ along $f: C \rightarrow$ $\mathscr{D}(X)$ is given by taking $(X \cdot f)(P)=C(P)$ for all $P \in \mathbb{C}_{0}$ and, for all transition types $P+Q \rightarrow L,(X \cdot f)(L)$ to be the pullback

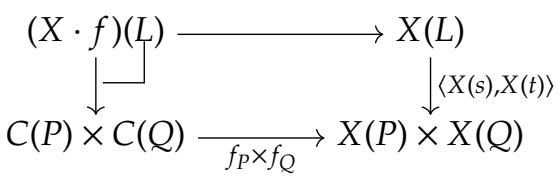

Corollary 4.5. Any morphism in $\mathscr{C}$ factors as a vertical morphism $l(=$ such that $\mathscr{D}(l)=i d)$, followed by a cartesian one.

\subsection{Substitution-closed bisimulation}

With the setting of Howe contexts in place, we now abstract over substitution-closed bisimulations.

Plain, functional bisimulations are defined by lifting against all maps $s$ from distinguished copairings $[s, t]$, e.g., as in (6). Similarly, bisimulation relations are defined as relations whose projections are functional bisimulations.
Now, substitution-closed bisimulations should live in a category of transition systems whose underlying object in $\mathscr{C}_{0}$ is substitution-closed. As we will use this idea of transition systems with structured underlying object several times, let us factor out the construction:

Lemma 4.6. For any monadic functor $U_{0}: \mathscr{E} \rightarrow \mathscr{C}_{0}$, consider the pullback

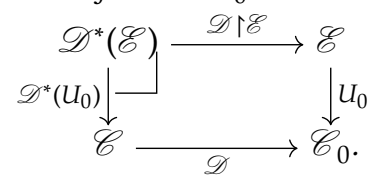

If the monad induced by $U_{0}$ is accessible, then $\mathscr{D} \uparrow \mathscr{E}$ has a fully-faithful left adjoint and $\mathscr{D}^{*}\left(U_{0}\right)$ has a left adjoint.

Terminology 4.7. If objects of $\mathscr{E}$ are called a certain name, say, things, then objects of $\mathscr{D}^{*}(\mathscr{E})$ will often be called transition things.

Definition 4.8. Let transition monoids be the objects of $\operatorname{Mon}(\mathscr{C}):=\mathscr{D}^{*}(\operatorname{mon}(\mathscr{C}))$, where $\operatorname{mon}(\mathscr{C})$ is the category of monoids in $\mathscr{C}_{0}$.

Furthermore, let the category of transition $X$-modules be $X-\operatorname{Mod}:=\mathscr{D}^{*}(\mathscr{D}(X)$-mod $)$, for any $X \in \operatorname{Mon}(\mathscr{C})$.

We let $\mathscr{F}: \mathscr{D}(X)$-mod $\rightarrow \mathscr{C}_{0}$ denote the forgetful functor, and $\mathscr{F}^{\prime}=\mathscr{D}^{*}(\mathscr{F}): X-\operatorname{Mod} \rightarrow \mathscr{C}$.

So transition $X$-modules are objects $M \in \mathscr{C}$ whose underlying $\mathscr{D}(M) \in \mathscr{C}_{0}$ is a $\mathscr{D}(X)$-module.

Definition 4.9. For any transition monoid $X$, a functional $X$-bisimulation is a map of transition $X$-modules whose image under $\mathscr{F}^{\prime}$ is a functional bisimulation. An $X$-bisimulation is a relation of transition $X$-modules whose projections are both functional $X$-bisimulations.

We are now interested in defining the largest $X$-bisimulation. This requires the following few intermediate results, leading to Corollary 4.12.

Proposition 4.10. The forgetful functor $\mathscr{F}^{\prime}: X-\operatorname{Mod} \rightarrow \mathscr{C}$ creates unions.

Lemma 4.11. X-bisimulations are closed under unions.

Proof. X-bisimulations are in particular plain bisimulations, so any union, which is computed as in $\mathscr{C}$ by Proposition 4.10, is again a bisimulation, as desired.

Corollary 4.12. For any $X$, the union $\sim_{X}^{\otimes}$ of all $X$-bisimulations over $X$, called $X$-bisimilarity, is an X-bisimulation.

\subsection{Signatures, models, and initiality}

In this section, in order to specify syntax and transition rules in the abstract setting, we introduce the notion of 2-signature and its models. We furthermore show that under mild hypotheses any 2-signature admits free models.

Let us first incorporate syntax into the transition monoids of Definition 4.8. For a general Howe context and structurally strong endofunctor $\Sigma_{0}$ on $\mathscr{C}_{0}$, we first define the category 
$\Sigma_{0}$-Mon $:=\mathscr{D}^{*}\left(\Sigma_{0}\right.$-mon $)$ of transition $\Sigma_{0}$-monoids with notation as below left.

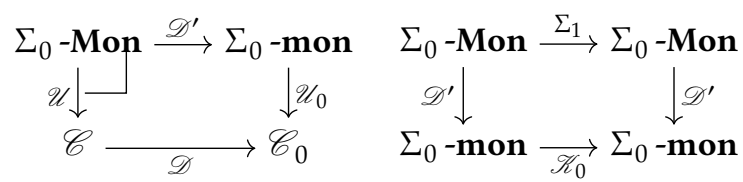

Notation 4.13. We often omit $\mathscr{D}^{\prime}$ and $\mathscr{U}$ for readability.

An endofunctor $\Sigma_{1}$ on $\Sigma_{0}$-Mon will be called syntactically free iff the $\Sigma_{0}$-monoid structure of any $\mathscr{D}^{\prime}\left(\Sigma_{1}(X)\right)$ is precisely the free structure on $\mathscr{U}_{0}\left(\mathscr{D}^{\prime}(X)\right)$, i.e., $\mathscr{D}^{\prime}\left(\Sigma_{1}(X)\right)=$ $\mathscr{L}_{0}\left(\mathscr{U}_{0}\left(\mathscr{D}^{\prime}(X)\right)\right)$, where $\mathscr{L}_{0}$ denotes the left adjoint to $\mathscr{U}_{0}$. Otherwise said, letting $\mathscr{K}_{0}$ denote the induced comonad $\mathscr{L}_{0}$ 。 $\mathscr{U}_{0}$, the diagram above right commutes.

Notation 4.14. Let $\mathscr{T}_{0}=\mathscr{U}_{0} \circ \mathscr{L}_{0}$, the induced monad.

Definition 4.15. A 2-signature on a Howe context $\mathbb{C} \rightarrow$ 2 consists of a structurally strong, finitary endofunctor $\Sigma_{0}$ on $\mathscr{C}_{0}$ preserving wide pullbacks, and a syntactically free endofunctor $\Sigma_{1}$ on transition $\Sigma_{0}$-monoids.

Remark 4.16. Any 2-signature induces a signature in the sense of Hirschowitz et al. [20, §4.1].

Let us now define the category of models of a 2-signature, by mimicking the concrete notion of vertical algebra from §3.2. For this, we observe that passing from $\Sigma_{1}^{\Lambda}$ to $\check{\Sigma}_{1}^{\Lambda}$ may be done by opcartesian lifting in the sense of Lemma 4.4. Indeed, bifibrations are stable under pullback, so $\mathscr{D}^{\prime}$ is again a bifibration, and we may state:

Definition 4.17. Let $\check{\Sigma}_{1}: \Sigma_{0}$-Mon $\rightarrow \Sigma_{0}$-Mon be defined by letting $\check{\Sigma}_{1}(X)$ be a choice of oplifting of $\Sigma_{1}(X)$ along the counit $\varepsilon_{\mathscr{D}^{\prime}(X)}^{0}: \mathscr{D}^{\prime}\left(\Sigma_{1}(X)\right)=\mathscr{K}_{0}\left(\mathscr{D}^{\prime}(X)\right) \rightarrow \mathscr{D}^{\prime}(X)$.

We obtain by construction:

Proposition 4.18. The triangle below commutes.

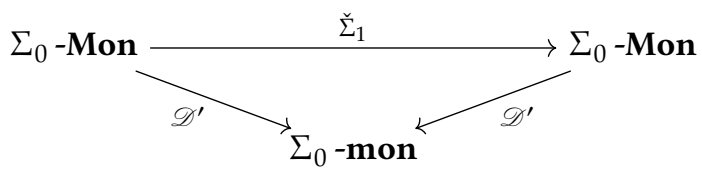

Definition 4.19. Let the category $\check{\Sigma}_{1}-\operatorname{alg}_{v}$ be the full subcategory of $\check{\Sigma}_{1}$-alg on vertical $\check{\Sigma}_{1}$-algebras, i.e., ones whose structure maps $\check{\Sigma}_{1}(X) \rightarrow X$ are sent by $\mathscr{D}^{\prime}$ to identities.

The functor $\check{\Sigma}_{1}$ restricts to an endofunctor $\left(\check{\Sigma}_{1}\right)_{\mid X}$ on the fibre over any $\Sigma_{0}$-monoid $X$, and we call $\Sigma_{1}$ vertically finitary iff each such restriction $\left(\check{\Sigma}_{1}\right)_{\mid X}$ is finitary.

Theorem 4.20. If $\Sigma_{1}$ is vertically finitary, then vertical $\check{\Sigma}_{1}$ algebras are monadic over transition $\Sigma_{0}$-monoids, and the free vertical $\check{\Sigma}_{1}$-algebra over any given $X$ is $\mu A .\left(X+\left(\check{\Sigma}_{1}\right)_{\mid \mathscr{D}^{\prime}(X)}(A)\right)$, i.e., colim $_{n \in \omega} X_{n}$ with $X_{0}=X$ and $X_{n+1}=X+\left(\check{\Sigma}_{1}\right)_{\mid \mathscr{X}^{\prime}(X)}\left(X_{n}\right)$. In particular, the initial vertical $\check{\Sigma}_{1}$-algebra is the colimit (in the fibre) of the chain $0^{\circledast} \rightarrow\left(\check{\Sigma}_{1}\right)_{\mid 0^{\circledast}}\left(0^{\circledast}\right) \rightarrow\left(\check{\Sigma}_{1}\right)_{\mid 0^{\circledast}}^{2}\left(0^{\circledast}\right) \rightarrow$... where $0^{\circledast}$ denotes the initial $\Sigma_{0}$-monoid.

\subsection{Weak compositionality}

We conclude this section by showing weak compositionality of the initial vertical algebra, say $\mathbf{Z}=\mu A \cdot\left(\check{\Sigma}_{1}\right)_{\mid 0 \circledast}(A)$, under a suitable hypothesis. The only thing we will need to know is that the source term of a transition in $\Sigma_{1}(X)$ has depth one. In order to state this properly, let us observe that by (12) we have $\mathscr{D} \mathscr{U} \Sigma_{1}=\mathscr{T}_{0} \mathscr{D} \mathscr{U}$, so by universal property of counit, as $\mathscr{D}(\mathscr{M}(P)) \cong P$, for any transition $r: L \rightarrow \mathscr{U}\left(\Sigma_{1}(X)\right)$, the source $r \circ s$ admits a unique lifting $r \backslash s$ as shown below.

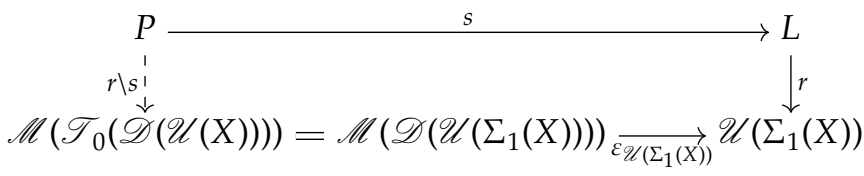

Definition 4.21. The 2-signature $\left(\Sigma_{0}, \Sigma_{1}\right)$ is layered iff for any $r: L \rightarrow \mathscr{U}\left(\Sigma_{1}(X)\right), r \backslash s$ lifts through $\mathscr{M}\left(\eta_{\Sigma_{0}, \mathscr{D}(\mathscr{U}(X))}\right)$, as in

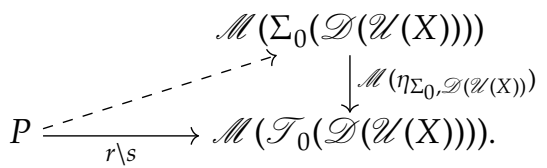

Informally, this means that the source of the conclusion of a transition rule must be an operation applied to some "metavariables": given any transition $r \in \Sigma_{1}(X)(L)$, there exists $m \in \Sigma_{0}(X)(P)$ such that $r \cdot s=\eta(m)$, with $\eta: \Sigma_{0} \rightarrow \mathscr{T}_{0}$.

Remark 4.22. As $\eta$ is monic by Theorem 2.19 , and $\mathscr{M}$ preserves monos, the lifting is unique.

Proposition 4.23. If $\left(\Sigma_{0}, \Sigma_{1}\right)$ is layered, then any commuting square as the solid part below admits a lifting $k$ as shown.

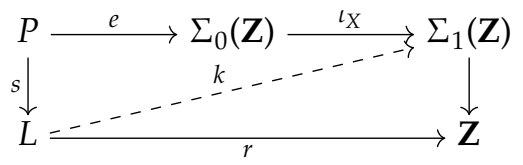

\subsection{Congruence statement}

We have now characterised the initial vertical algebra for a 2-signature as our abstract model of syntactic transition systems from operational semantics. Let us now state the desired congruence result in this abstract setting.

Definition 4.24. A relation $R \hookrightarrow \mathscr{U}(X)^{2}$ over a transition $\Sigma_{0}$-monoid $X$ is a congruence iff it is an equivalence relation and furthermore, omitting $\mathscr{U}$, the composite

$$
\mathscr{T}_{0}(\mathscr{D}(R)) \rightarrow \mathscr{T}_{0}\left(\mathscr{D}\left(X^{2}\right)\right) \rightarrow \mathscr{T}_{0}(\mathscr{D}(X))^{2} \rightarrow \mathscr{D}(X)^{2}
$$

factors through $\mathscr{D}(R) \rightarrow \mathscr{D}(X)^{2}$.

Let us directly reduce this to the following easier form:

Lemma 4.25. An equivalence relation $R \hookrightarrow \mathscr{U}(X)^{2}$ is a congruence iff $\mathscr{D}(R)$ is both a submonoid and a subalgebra of $\mathscr{D}(\mathscr{U}(X))^{2}$, i.e., omitting $\mathscr{U}$ and $\mathscr{D}$, both composites

$$
\begin{array}{r}
R \otimes R \rightarrow X^{2} \otimes X^{2} \rightarrow(X \otimes X)^{2} \rightarrow X^{2} \\
\Sigma_{0}(R) \rightarrow \Sigma_{0}\left(X^{2}\right) \rightarrow \Sigma_{0}(X)^{2} \rightarrow X^{2}
\end{array}
$$


factor through $R \rightarrow X^{2}$.

\section{Howe's method}

In the previous section, we have introduced 2-signatures and shown that under suitable hypotheses they admit initial models (= initial vertical algebras) which are weakly compositional. We now want to adapt Howe's method to this abstract setting and show that substitution-closed bisimilarity on the initial vertical algebra is a congruence. We thus fix any vertically finitary, layered 2 -signature $\left(\Sigma_{0}, \Sigma_{1}\right)$, and let $\mathbf{Z}$ denote its initial vertical algebra. We start in $\S 5.1$ by giving a brief introduction to Howe's method. In $\S 5.2$, we continue with some preparatory work: we introduce prebisimulation, which is very close to lifting-based bisimulation, except that, unlike for plain bisimulations, it makes sense to take the context closure of a prebisimulation. We exploit this in §5.3, where we introduce our abstract version of the Howe closure $R^{\bullet}$ of a relation $R$. We furthermore reduce congruence of bisimilarity $\sim_{\mathbf{Z}}^{\otimes}$ for the initial vertical $\Sigma_{1}$-algebra $\mathbf{Z}$ to the fact that the Howe closure $\left(\sim_{\mathbf{Z}}^{\vee, \otimes}\right)^{\bullet}$ of the associated prebisimulation is itself a presimulation. Finally, in $§ 5.4$, we introduce cospan-cellularity and show that if $\Sigma_{1}$ is cospancellular, then $\left(\sim_{\mathbf{Z}}^{\vee, \otimes}\right)^{\bullet}$ is indeed a presimulation, which entails the main result.

\subsection{A subjective introduction to Howe's method}

Let us start by briefly recalling Howe's method, loosely following Pitts [31]. Let us return to our running example, bigstep, pure $\lambda$-calculus, and naively attempt to prove that bisimilarity is context-closed. The idea is to consider some contextclosed relation $\sim_{\mathbf{Z}}^{\bullet}$, containing $\sim_{\mathbf{Z}}^{\otimes}$ by construction, and then to show that it is a bisimulation. By maximality of $\sim \sim_{\mathbf{Z}}^{\otimes}$, we then also have $\sim_{\mathbf{Z}}^{\dot{S}} \subseteq \sim_{\mathrm{Z}}^{\otimes}$ hence both relations coincide and $\sim_{\mathbf{Z}}^{\otimes}$ is context-closed as desired.

In order for $\sim_{\mathbf{Z}}^{\bullet}$ to be context-closed, it should at least contain all pairs $\left(C\left[e_{1}, \ldots, e_{n}\right], C\left[e_{1}^{\prime}, \ldots, e_{n}^{\prime}\right]\right)$ with $e_{i} \sim_{\mathbf{Z}}^{\otimes} e_{i}^{\prime}$ for all $i \in n$. Typically, let us consider $e_{1} \sim_{\mathbf{Z}}^{\otimes} e_{1}^{\prime}$ and $e_{2} \sim_{\mathbf{Z}}^{\otimes} e_{2}^{\prime}$, and try to prove the simulation property for $e_{1} e_{2} \sim_{\mathbf{Z}} e_{1}^{\prime} e_{2}^{\prime}$. We thus assume $e_{1} \Downarrow \lambda x . e_{3}$ and $e_{3}\left[e_{2}\right] \Downarrow e_{4}$, and try to find $e_{3}^{\prime}$ such that $e_{1}^{\prime} \Downarrow \lambda x . e_{3}^{\prime}$ and $e_{4}^{\prime}$ such that $e_{3}^{\prime}\left[e_{2}^{\prime}\right] \Downarrow e_{4}^{\prime}$ with $e_{4} \sim_{\mathbf{Z}} e_{4}^{\prime}$. Because $e_{1} \sim_{\mathbf{Z}}^{\otimes} e_{1}^{\prime}$, we find $e_{1}^{\prime} \Downarrow \lambda x . e_{3}^{\prime}$ such that for all $e, e_{3}[e] \sim_{\mathrm{Z}}^{\otimes} e_{3}^{\prime}[e]$. This $e_{3}^{\prime}$ is a natural candidate to suit our needs. But then how do we find $e_{4}^{\prime}$ ? Because $\sim_{\mathrm{Z}}^{\bullet}$ is contextclosed and $\sim_{\mathrm{Z}}^{\otimes}$ is substitution-closed, we have

$$
e_{3}\left[e_{2}\right] \sim \sim_{\mathbf{Z}} e_{3}\left[e_{2}^{\prime}\right] \sim \sim_{\mathbf{Z}}^{\otimes} e_{3}^{\prime}\left[e_{2}^{\prime}\right] .
$$

Assuming we are reasoning by induction over the considered transition proof, we find $e_{4}^{\prime \prime}$ (by induction hypothesis) and $e_{4}^{\prime}$ (by definition of bisimulation) as in

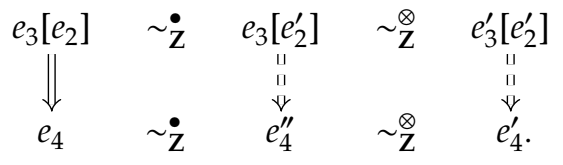

This suggests that $\sim_{\mathbf{Z}}$ should be closed under action of $\sim_{\mathbf{Z}}^{\otimes}$, i.e., $\sim_{\mathbf{Z}}^{\bullet} \sim_{\mathbf{Z}}^{\otimes} \subseteq \sim_{\mathbf{Z}}^{\bullet}$, or otherwise said: for all $e \sim_{\mathbf{Z}}^{\bullet} e^{\prime} \sim_{\mathbf{Z}}^{\otimes} e^{\prime \prime}$ we have $e \sim_{\mathbf{Z}} e^{\prime \prime}$. Howe's idea is to take this as a defining property of $\sim{ }_{\mathbf{Z}}^{\bullet}$. Coupling this with context closure, we define $\sim_{\mathbf{Z}}^{\bullet}$ as the smallest context-closed relation satisfying the rules

$$
\overline{x \sim_{\mathbf{Z}} x} \quad \frac{e \sim_{\mathbf{Z}} e^{\prime} e^{\prime} \sim \underset{\mathbf{Z}}{\otimes} e^{\prime \prime}}{e \sim \sim_{\mathbf{Z}} e^{\prime \prime}} .
$$

By construction, $\sim \dot{\mathrm{Z}}$ is reflexive and context-closed. By reflexivity and the second rule, it also contains $\sim_{\mathbf{Z}}^{\otimes}$.

Remark 5.1. In $\S 5.3$, we use an equivalent (because $\sim_{\mathbf{Z}}^{\otimes}$ is reflexive and transitive), inductive, and perhaps more compact definition.

The initial plan was to show that $\sim_{\mathbf{Z}}$ is a bisimulation and deduce that it coincides with $\sim_{\mathbf{Z}}^{\otimes}$. We can in fact optimise this slightly by first showing that $\sim_{\mathbf{Z}}^{\bullet}$ is a simulation, and then that its transitive closure $\left(\sim_{\mathbf{Z}}^{-}\right)^{+}$is symmetric. The relation $\left(\sim_{\mathbf{Z}}\right)^{+}$is thus a symmetric simulation, hence a bisimulation. This entails the last inclusion in the chain $\sim_{\mathbf{Z}}^{\otimes} \subseteq \sim_{\mathbf{Z}} \subseteq$ $(\sim \dot{\mathbf{Z}})^{+} \subseteq \sim \sim_{\mathbf{Z}}^{\otimes}$, showing that all relations coincide. Finally, because $\sim_{\mathbf{Z}}$ is context-closed, so is $\sim_{\mathbf{Z}}^{\otimes}$, as desired.

\subsection{Prebisimulations}

In order to adapt Howe's method to the abstract setting, we often need to consider the context closure $\Sigma_{0}(R)$ of relations, which lives in $\mathscr{C}_{0}$, not $\mathscr{C}$. It is thus more convenient to resort to the following variant of bisimulations, adapted from [21].

Definition 5.2. For any $X, Y \in \mathscr{C}$ and relations $R, R^{\prime} \subseteq$ $\mathscr{D}(X) \times \mathscr{D}(Y)$, we say that $R$ left-progresses to $R^{\prime}$, and write $R \sim_{l} R^{\prime}$, when any commuting square of the form below left, where $\mathscr{M}(R) \rightarrow X$ is obtained by transposition, may be embedded into some commuting diagram as below right.

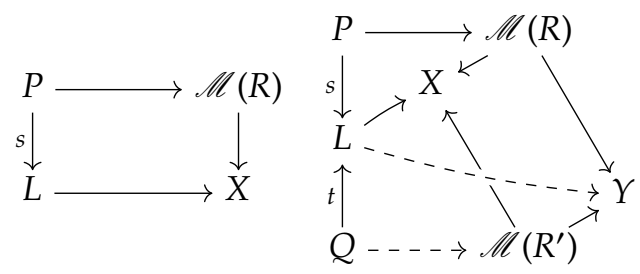

A presimulation is a relation $R$ such that $R \leadsto_{l} R$, and a prebisimulation is a presimulation whose converse relation is also a presimulation.

Intuitively, left-progression is one half of the standard notion of progression [33]: given any transition $x_{1} \stackrel{L}{\rightarrow} x_{2}$ with $x_{1} R y_{1}$, we find a transition $y_{1} \stackrel{L}{\rightarrow} y_{2}$ such that $x_{2} R^{\prime} y_{2}$.

Remark 5.3. This is equivalent to [21, Definition 5.1].

The advantage of prebisimulations over bisimulations is that they live in $\mathscr{C}_{0}$, hence morally only involve terms. However, they tell us essentially the same thing as bisimulations, 
as shown in $[21, \S 5.2]$. In particular, one can prove that prebisimulations are closed under union.

Definition 5.4. The union of all prebisimulations over any fixed $X$ is called prebisimilarity and denoted by $\sim \underset{X}{\vee}$.

Furthermore, this lifts to substitution-closed prebisimulations: let an $X$-prebisimulation be a relation $R \hookrightarrow \mathscr{D}(M)^{2}$ in $X$-mod, for any $M \in X$-Mod, such that $\mathscr{F}(R)$ is a prebisimulation; we have that $X$-prebisimulations are closed under unions, whence:

Definition 5.5. Let $\stackrel{\vee}{\sim}{ }_{X}^{\vee}$ denote the union of all $X$-prebisimulations over $X$, called $X$-prebisimilarity.

Proposition 5.6. We have $\mathscr{D}\left(\sim_{X}^{\otimes}\right)=\sim_{X}^{\vee}, \otimes$.

\subsection{Howe closure}

We now want to prove that $\sim_{\mathbf{Z}}^{\otimes}$ is a congruence, where, we recall, $\mathbf{Z}=\mu A .\left(\check{\Sigma}_{1}\right)_{\mid 0^{\circledast}}(A)$ is the initial vertical algebra.

Lemma 5.7. If $\sim_{\mathbf{Z}}^{\vee, \otimes}$ is both a subalgebra and a submonoid of $\mathscr{D}(\mathbf{Z})^{2}$, then $\sim_{\mathbf{Z}}^{\otimes}$ is a congruence.

Proof. We have $\mathscr{D}\left(\sim_{\mathbf{Z}}^{\otimes}\right)=\sim_{\mathbf{Z}}^{\vee}, \otimes$ by Proposition 5.6, so we conclude by Lemma 4.25

So we need to prove that $\sim_{\mathbf{Z}}^{\vee, \otimes}$ is both a subalgebra and a submonoid. For this, we use the following variant of Howe's construction.

Definition 5.8. For any relations $R$ and $A$ on $\mathscr{D}(\mathscr{U}(X))$, let $\mathscr{H}_{R}(A)$ denote the image (in $\mathscr{C}_{0}$ ) of $I+\Sigma_{0}(A) ; R$ in $X^{2}$, where $I$ is viewed as a relation through the unit and diagonal maps $I \rightarrow X \rightarrow X^{2}$ and ; denotes sequential composition of relations. The Howe closure $R^{\bullet}$ of any $R$ is the initial $\mathscr{H}_{R^{-}}$ algebra, i.e., the (directed) union $\bigcup_{n} \mathscr{H}_{R}^{n}(\varnothing)$.

Let us first show the following lemma, in case $X=\mathbf{Z}$.

Lemma 5.9. If $R \subseteq \mathbf{Z}^{2}$ is reflexive, transitive, and a $\mathbf{Z}$-module, then (omitting im(-) for readability)

(i) $\mathbf{Z} \subseteq R^{\bullet}$ (and so $I \subseteq R^{\bullet}$ );

(ii) $R^{\bullet} ; R \subseteq R^{\bullet}$ (and so $R \subseteq R^{\bullet}$ by (i));

(iii) $\Sigma_{0}\left(R^{\bullet}\right) \subseteq R^{\bullet}$;

(iv) $R^{\bullet} \otimes R^{\bullet} \subseteq R^{\bullet}$ (and so $R^{\bullet} \otimes \mathbf{Z} \subseteq R^{\bullet}$ by (i)).

Corollary 5.10. We have $\mathscr{T}_{0}\left(R^{\bullet}\right) ; R \subseteq R^{\bullet}$.

We then narrow down the goal as follows, using $(-)^{+}$to denote transitive closure.

Lemma 5.11. If $\left(\sim_{\mathbf{Z}}^{\vee, \otimes}\right)^{\bullet+}$ is a prebisimulation, then $\sim_{\mathbf{Z}}^{\otimes}$ is a congruence.

Proof. By hypothesis, $\left(\sim_{\mathbf{Z}}^{\vee, \otimes}\right)^{\bullet+}$ is a prebisimulation, hence $\left(\sim_{\mathbf{Z}}^{\vee, \otimes}\right)^{\bullet+} \subseteq \sim_{\mathbf{Z}}^{\vee, \otimes}$. Moreover, the converse also holds by Lemma $5.9(i i)$, so $\left(\sim_{\mathbf{Z}}^{\vee} \otimes\right)^{\bullet+}=\sim_{\mathbf{Z}}^{\vee, \otimes}$. But by Lemma 5.9 and the fact that subalgebras and submonoids are stable under transitive closure, $\left(\sim_{\mathbf{Z}}^{\vee, \otimes}\right)^{\bullet+}$ is a subalgebra and a submonoid, hence so is $\sim_{\mathbf{Z}}^{\vee, \otimes}$, and we conclude by Lemma 5.7.
This would leave us with the task of delineating hypotheses under which $\left(\sim_{\mathbf{Z}}^{\vee, \otimes}\right)^{\bullet+}$ is indeed a prebisimulation, but we can narrow this down a bit further (this is sometimes called the transitive closure trick), using the following two intermediate lemmas.

Lemma 5.12. If $R \subseteq X^{2}$ in $\mathscr{C}$ is a presimulation up to transitivity [33], i.e., $R \leadsto_{l} R^{+}$, then $R^{+}$is a presimulation.

Lemma 5.13. If $R \subseteq \mathbf{Z}^{2}$ is an equivalence relation, then so is $R^{\bullet+}$.

Lemma 5.14. If $\left(\sim_{\mathrm{Z}}^{\vee, \otimes}\right)^{\bullet}$ is a presimulation, then $\sim_{\mathrm{Z}}^{\otimes}$ is a congruence.

Proof. By Lemma 5.12, $\left(\sim_{\mathbf{Z}}^{\vee, \otimes}\right)^{\bullet+}$ is a presimulation. But $\left(\sim_{\mathbf{Z}}^{\vee, \otimes}\right)^{\bullet+}$ is symmetric by Lemma 5.13 , so it is in fact a prebisimulation, hence we conclude by Lemma 5.11 .

\subsection{Familiality and cellularity}

In the previous section, we have established that congruence of $\sim_{\mathbf{Z}}^{\otimes}$ will follow from $\left(\sim_{\mathbf{Z}}^{\vee, \otimes}\right)^{\bullet}$ being a presimulation, and so are now seeking sufficient conditions for this. Our key tool will be cellularity, a special case of familiality. As we have already seen that $\Sigma_{0}^{\Lambda}$ is familial (Proposition 2.6), let us now start by proving that $\Sigma_{1}^{\Lambda}$ is so too.

Example 5.15. $\Sigma_{1}^{\Lambda}$ is familial, and $\Sigma_{0}^{\Lambda} \rightarrow \Sigma_{1}^{\Lambda}$ preserves generics. Using Theorem 2.5, the interesting point is that we have $\Sigma_{1}^{\Lambda}(X)(\Downarrow) \cong\left[\mathbf{y}_{1}, X\right]+\left[E_{\beta}, X\right]$, where $E_{\beta}$ denotes the colimit of the diagram

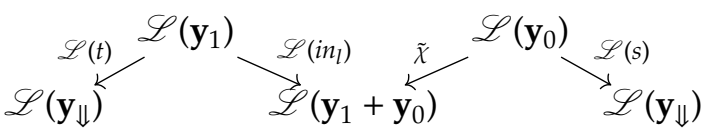

with $\tilde{\chi}$ denoting the transpose of the morphism $\chi: \mathbf{y}_{0} \rightarrow$ $\mathscr{U} \mathscr{L}\left(\mathbf{y}_{1}+\mathbf{y}_{0}\right)$ corresponding by Yoneda to the element

$$
\left(\operatorname{in}_{l}\left(i d_{1}\right)\right)\left(i n_{r}\left(i d_{0}\right)\right) \in \mathscr{U} \mathscr{L}\left(\mathbf{y}_{1}+\mathbf{y}_{0}\right)(0) .
$$

On the other hand, $\check{\Sigma}_{1}$ is not familial, because the candidate assignment $\operatorname{el}\left(\check{\Sigma}_{1}(1)\right) \rightarrow \mathscr{C}$ is not functorial.

Howe's approach to proving that $\left(\sim_{\mathbf{Z}}^{\vee, \otimes}\right)^{\bullet}$ is a presimulation is by induction on the given transition. More generally, for any prebisimulation $R$ satisfying the hypotheses of Lemma 5.9, we prove by induction on $n$ that any commuting diagram as the solid part below left

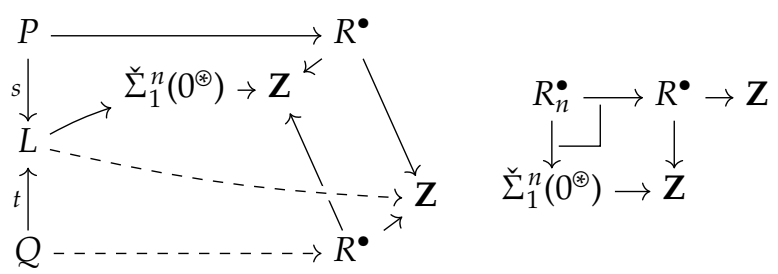

may be completed as shown. This indeed entails that $R^{\bullet}$ is a presimulation, because $\mathbf{Z}$ is the colimit of all $\check{\Sigma}_{1}^{n}\left(0^{\circledast}\right)$, any 
morphism $L \rightarrow \mathbf{Z}$ factors through some $\check{\Sigma}_{1}^{n}\left(0^{\circledast}\right) \rightarrow \mathbf{Z}$, hence we get $R^{\bullet} \sim_{l} R^{\bullet}$ as desired. But this is equivalent to the relation $R_{n}^{\bullet} \hookrightarrow \check{\Sigma}_{1}^{n}\left(0^{\circledast}\right) \times \mathbf{Z}$ obtained by pullback above right is a presimulation, so we want to prove by induction that each $R_{n}^{\bullet}$ is a presimulation.

Let us isolate the interesting part of this inductive proof:

Lemma 5.16. For all $n>0, R_{n}^{\bullet}$ is a presimulation if, for all commuting diagrams

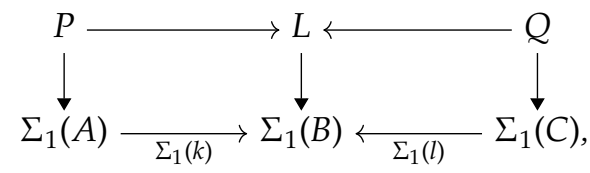

where $\rightarrow$ denotes generic morphisms, $k$ and $l$ are such that any diagram of the form below left may be completed as shown.
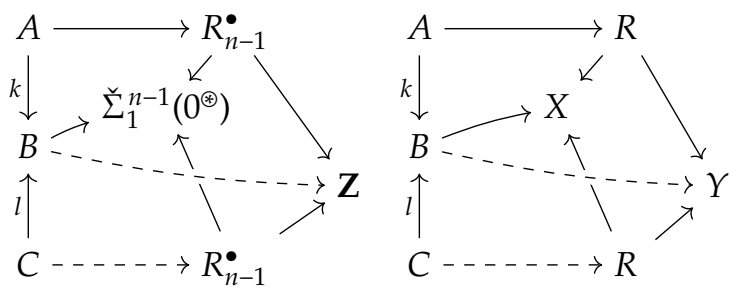

By induction hypothesis, we know that $R_{n-1}^{\bullet}$ is a presimulation, but the problem is that $(k, l)$ may not be of the form $(s, t)$, and will rarely be so in practice. This is precisely where cellularity comes into play.

Definition 5.17. A copresimulation is any cospan $(k, l)$ such that for any presimulation $R \hookrightarrow X \times Y$, any square as in (19, right) may be completed as shown.

The functor $\Sigma_{1}$ is cospan-cellular iff it is familial and, for all commuting diagrams (18) with generic vertical arrows, the cospan $(k, l)$ is a copresimulation.

If $\Sigma_{1}$ is cospan-cellular, then by induction hypothesis $R_{n-1}^{\bullet}$ is a presimulation, so (19, left) may be completed as shown, so by Lemma $5.16 R_{n}^{\bullet}$ is a presimulation. We have proved:

Lemma 5.18. If $\Sigma_{1}$ is cospan-cellular and $R \subseteq \mathbf{Z}^{2}$ is a prebisimulation satisfying the hypotheses of Lemma 5.9, the relation $R^{\bullet}$ is a presimulation.

Thus, by Lemma 5.14, we obtain:

Theorem 5.19. For any vertically finitary, layered 2-signature $\left(\Sigma_{0}, \Sigma_{1}\right)$ with $\Sigma_{1}$ cospan-cellular, substitution-closed bisimilarity $\sim_{\mathbf{Z}}^{\otimes}$ on the initial vertical algebra is a congruence.

A mysterious bit remains: what does cospan-cellularity mean? In order to demonstrate that our running example $\Sigma_{1}^{\Lambda}$ is cospan-cellular, we first need to develop some basic results about copresimulations.

Lemma 5.20. Copresimulations contain the basic cospans $(s, t)$ and identity cospans. Moreover, they are closed under pointwise coproduct, precomposition of their right-hand leg with any morphism, and cospan composition.
Example 5.21. Recalling Example 5.15, we need to show that the cospan $\mathscr{L}\left(\mathbf{y}_{0}+\mathbf{y}_{0}\right) \rightarrow E_{\beta} \leftarrow \mathscr{L}\left(\mathbf{y}_{1}\right)$ corresponding to the $\beta$-rule is a copresimulation, which holds by Lemma 5.20 , as it is the composite of

$$
\mathscr{L}\left(\mathbf{y}_{0}+\mathbf{y}_{0}\right) \rightarrow \mathscr{L}\left(\mathbf{y}_{\Downarrow}+\mathbf{y}_{0}\right) \leftarrow \mathscr{L}\left(\mathbf{y}_{1}+\mathbf{y}_{0}\right) \stackrel{\tilde{\chi}}{\leftarrow} \mathscr{L}\left(\mathbf{y}_{0}\right)
$$

and the basic cospan $\mathscr{L}\left(\mathbf{y}_{0} \rightarrow \mathbf{y}_{\Downarrow} \leftarrow \mathbf{y}_{1}\right)$.

Example 5.22. The call-by-value $\lambda$-calculus may be treated similarly, with the same $\Sigma_{0}$, using the same rule for values (5, right), but changing rule (5, left) to

$$
\frac{e_{1} \Downarrow e_{1}^{\prime} \quad e_{2} \Downarrow e_{2}^{\prime} \quad e_{1}^{\prime}\left[\lambda . e_{2}^{\prime}\right] \Downarrow e_{3}}{e_{1} e_{2} \Downarrow e_{3}} .
$$

The induced functor $\Sigma_{1}$ is cospan-cellular by Lemma 5.20 , since the cospan (18) for the new rule may be obtained by starting with the pointwise coproduct

$$
\mathscr{L}\left(2 \cdot \mathbf{y}_{0}\right) \stackrel{\mathscr{L}(2 \cdot s)}{\longrightarrow} \mathscr{L}\left(2 \cdot \mathbf{y}_{\Downarrow}\right) \stackrel{\mathscr{L}(2 \cdot t)}{\longleftarrow} \mathscr{L}\left(2 \cdot \mathbf{y}_{1}\right)
$$

(where $n \cdot c$ denotes the $n$-fold coproduct $c+\ldots+c$ ), precomposing its right-hand leg with

$$
\mathscr{L}\left(\mathbf{y}_{0}\right) \stackrel{\tilde{x}}{\rightarrow} \mathscr{L}\left(\mathbf{y}_{1}\right)+\mathscr{L}\left(\mathbf{y}_{0}\right) \stackrel{\mathscr{L}\left(\mathbf{y}_{1}\right)+\tilde{\lambda}}{\longrightarrow} 2 \cdot \mathscr{L}\left(\mathbf{y}_{1}\right) \cong \mathscr{L}\left(2 \cdot \mathbf{y}_{1}\right),
$$

and then composing with the basic cospan.

\section{Conclusion and perspectives}

We have presented an abstract framework, 2-signatures on Howe contexts, in which, under suitable hypotheses, substitution-closed bisimilarity is a congruence. We have proved new initiality and familiality results along the way, notably an adaptation of work by Fiore and collaborators to the skewmonoidal case. Finally, we have covered the basic examples of call-by-name and call-by-value variants of big-step $\lambda$-calculus. Future research directions include trying to generalise our framework to cover variants of Howe's method which currently seem to lie beyond its scope, e.g., so-called early-style bisimilarity in higher-order $\pi$-calculus or calculi with passivation [27]. Similarly, we plan to try and apply our techniques to variants of applicative bisimilarity, e.g., open bisimilarity [26].

\section{Acknowledgments}

Thanks to Marcelo Fiore, Richard Garner, Shin-ya Katsumata, Sergueï Lenglet, Alan Schmitt, Pawel Sobocinski, Sam Staton, and the anonymous referees for useful input.

This research has partly been funded by the CoqHoTT ERC Grant 637339.

\section{References}

[1] Martín Abadi, Luca Cardelli, Pierre-Louis Curien, and Jean-Jacques Lévy. 1990. Explicit Substitutions. In Proc. 17th International Symposium on Principles of Programming Languages, Frances E. Allen (Ed.). ACM, 31-46. https://doi.org/10.1145/96709.96712 
[2] Samson Abramsky. 1990. The lazy lambda calculus. In Research Topics in Functional Programming, D. A. Turner (Ed.). Addison-Wesley.

[3] Jiří Adámek and Jiří Rosicky. 1994. Locally Presentable and Accessible Categories. Cambridge University Press. https://doi.org/10.1017/ CBO9780511600579

[4] Benedikt Ahrens, André Hirschowitz, Ambroise Lafont, and Marco Maggesi. 2019. Reduction monads and their signatures. PACMPL 4, POPL (2019). https://doi.org/10.1145/3371099

[5] Thorsten Altenkirch, James Chapman, and Tarmo Uustalu. 2015. Monads need not be endofunctors. Logical Methods in Computer Science 11, 1 (2015). https://doi.org/10.2168/LMCS-11(1:3)2015

[6] Martin Bodin, Philippa Gardner, Thomas Jensen, and Alan Schmitt. 2019. Skeletal semantics and their interpretations. PACMPL 3, POPL (2019), 44:1-44:31. https://doi.org/10.1145/3290357

[7] Filippo Bonchi, Daniela Petrisan, Damien Pous, and Jurriaan Rot. 2014. Coinduction up-to in a fibrational setting. In Proc. 29th Symposium on Logic in Computer Science ACM, 20:1-20:9. https://doi.org/10.1145/ 2603088.2603149

[8] A. K. Bousfield. 1977. Constructions of Factorization Systems in Categories. Journal of Pure and Applied Algebra 9, 2-3 (1977), 287-329.

[9] Aurelio Carboni and Peter Johnstone. 1995. Connected Limits, Familial Representability and Artin Glueing. Mathematical Structures in Computer Science 5, 4 (1995), 441-459. https://doi.org/10.1017/ S0960129500001183

[10] Aurelio Carboni, Stephen Lack, and Robert F. C. Walters. 1993. Introduction to extensive and distributive categories. Fournal of Pure and Applied Algebra 84, 2 (1993).

[11] Andrea Corradini, Reiko Heckel, and Ugo Montanari. 2002. Compositional SOS and beyond: a coalgebraic view of open systems. Theoretical Computer Science 280, 1-2 (2002), 163-192. https://doi.org/10. 1016/S0304-3975(01)00025-1

[12] Yves Diers. 1978. Spectres et localisations relatifs à un foncteur. Comptes rendus hebdomadaires des séances de l'Académie des sciences 287, 15 (1978), 985-988.

[13] Marcelo Fiore and Chung-Kil Hur. 2009. On the construction of free algebras for equational systems. Theoretical Computer Science 410 (2009), 1704-1729.

[14] Marcelo Fiore, Gordon Plotkin, and Daniele Turi. 1999. Abstract Syntax and Variable Binding. In Proc. 14th Symposium on Logic in Computer Science IEEE.

[15] Marcelo Fiore and Philip Saville. 2017. List Objects with Algebraic Structure. In Proc. 2nd International Conference on Formal Structures for Computation and Deduction (LIPIcs), Dale Miller (Ed.), Vol. 84. Schloss Dagstuhl - Leibniz-Zentrum fuer Informatik, 16:116:18. https://doi.org/10.4230/LIPIcs.FSCD.2017.16

[16] Marcelo Fiore and Philip Saville. 2019. List Objects with Algebraic Structure. (2019). Long version of [15].

[17] Marcelo P. Fiore. 2008. Second-Order and Dependently-Sorted Abstract Syntax. In Proc. 23rd Symposium on Logic in Computer Science IEEE, 57-68. https://doi.org/10.1109/LICS.2008.38

[18] Richard Garner and Tom Hirschowitz. 2018. Shapely monads and analytic functors. Journal of Logic and Computation 28, 1 (2018), 3383. https://doi.org/10.1093/logcom/exx029

[19] Makoto Hamana. 2004. Free S-Monoids: A Higher-Order Syntax with Metavariables (Lecture Notes in Computer Science), Wei-Ngan Chin (Ed.), Vol. 3302. Springer, 348-363. https://doi.org/10.1007/978-3-54030477-7_23

[20] André Hirschowitz, Tom Hirschowitz, and Ambroise Lafont. 2019. Modules over monads and operational semantics. (2019). https: //hal.archives-ouvertes.fr/hal-02338144 preprint.

[21] Tom Hirschowitz. 2019. Familial monads and structural operational semantics. PACMPL 3, POPL (2019), 21:1-21:28. https://dl.acm.org/ citation.cfm?id=3290334
[22] Mark Hovey. 1999. Model Categories. Mathematical Surveys and Monographs, Volume 63, AMS (1999), Vol. 63. American Mathematical Society.

[23] André Joyal, Mogens Nielsen, and Glynn Winskel. 1993. Bisimulation and open maps. In Proc. 8th Symposium on Logic in Computer Science IEEE, 418-427. https://doi.org/10.1109/LICS.1993.287566

[24] Stephen Lack and Ross Street. 2014. On monads and warpings. Cahiers de Topologie et Géométrie Différentielle Catégoriques LV, 4 (2014), 244-266.

[25] Stephen Lack and Ross Street. 2015. Skew-monoidal reflection and lifting theorems. Theory and Applications of Categories 30, 28 (2015), 985-1000.

[26] S. Lassen. 1999. Bisimulation in Untyped Lambda Calculus: Böhm Trees and Bisimulation up to Context. In Proc. 15th Conference on Mathematical Foundations of Progamming Semantics, MFPS 1999, Tulane University, New Orleans, LA, USA, April 28 - May 1, 1999 (Electronic Notes in Theoretical Computer Science), Stephen D. Brookes, Achim Jung, Michael W. Mislove, and Andre Scedrov (Eds.), Vol. 20. Elsevier, 346-374. https://doi.org/10.1016/S1571-0661(04)80083-5

[27] Sergueï Lenglet and Alan Schmitt. 2015. Howe's Method for Contextual Semantics. In Proc. 26th International Conference on Concurrency Theory, Luca Aceto and David de Frutos-Escrig (Eds.), Vol. 42. Schloss Dagstuhl - Leibniz-Zentrum fuer Informatik, 212-225. https: //doi.org/10.4230/LIPIcs.CONCUR.2015.212

[28] Saunders Mac Lane. 1998. Categories for the Working Mathematician (2nd ed.). Number 5 in Graduate Texts in Mathematics. Springer.

[29] Saunders Mac Lane and Ieke Moerdijk. 1992. Sheaves in Geometry and Logic: A First Introduction to Topos Theory. Springer.

[30] Robert Paré. 1990. Simply connected limits. Canadian fournal of Mathematics 42 (1990), 731-746.

[31] Andrew M. Pitts. 2011. Howe's method for higher-order languages, Chapter 5. In Sangiorgi and Rutten [35].

[32] Gordon D. Plotkin. 1981. A structural approach to operational semantics. DAIMI Report FN-19. Computer Science Department, Aarhus University.

[33] Damien Pous and Davide Sangiorgi. 2011. Enhancements of the bisimulation proof method, Chapter 6. In Sangiorgi and Rutten [35].

[34] Jan Reiterman. 1977. A left adjoint construction related to free triples. Journal of Pure and Applied Algebra 10 (1977), 57-71.

[35] Davide Sangiorgi and Jan Rutten (Eds.). 2011. Advanced Topics in Bisimulation and Coinduction. Number 52 in Cambridge Tracts in Theoretical Computer Science. Cambridge University Press.

[36] Sam Staton. 2008. General Structural Operational Semantics through Categorical Logic. In Proc. 23rd Symposium on Logic in Computer Science 166-177. https://doi.org/10.1109/LICS.2008.43

[37] Kornel Szlachányi. 2012. Skew-monoidal categories and bialgebroids. Advances in Mathematics 231 (2012), 1694-1730. https://doi.org/10. 1016/j.aim.2012.06.027

[38] Daniele Turi and Gordon D. Plotkin. 1997. Towards a Mathematical Operational Semantics. In Proc. 12th Symposium on Logic in Computer Science 280-291. https://doi.org/10.1109/LICS.1997.614955

[39] Vladimir Voevodsky, Benedikt Ahrens, Daniel Grayson, et al. 2019. UniMath - a computer-checked library of univalent mathematics. https://github.com/UniMath/UniMath

[40] Mark Weber. 2004. Generic morphisms, parametric representations and weakly cartesian monads. Theory and Applications of Categories 13 (2004), 191-234.

[41] Mark Weber. 2007. Familial 2-functors and parametric right adjoints. Theory and Applications of Categories 18, 22 (2007), 665-732. 


\section{A Syntax: familiality and substitution}

This section collects additional detail for $\$ 2$, aside from the proofs of both main theorems, which are dealt with in the next two sections.

Proof of Proposition 2.6. Consider the morphism $\mathbf{y}_{0} \rightarrow \mathscr{T}_{0}^{\Lambda}(1)$ picking the term $\square_{0}(\mid) \in \mathscr{T}_{0}^{\Lambda}(1)(0)$, where $1(n)=\mathbf{y}_{0}(n)=$ $\left\{\square_{0}\right\}$ for all $n$, and assume it factors as

$$
\mathbf{y}_{0} \stackrel{f}{\rightarrow} \mathscr{T}_{0}^{\Lambda}(A) \stackrel{\mathscr{T}_{0}^{\Lambda}(!)}{\longrightarrow} \mathscr{T}_{0}^{\Lambda}(1) .
$$

Then, in order for the composite to be $\square_{0}()$, we have $f=$ $o\left(e_{1}, \ldots, e_{n}\right)$, for some $o \in A(n)$ and $e_{1}, \ldots, e_{n} \in \mathscr{T}_{0}^{\Lambda}(A)(0)$. Let us show that this factorisation is not generic. For this, we observe that any term $e \in \mathscr{T}_{0}^{\Lambda}(0)(0)$ yields a factorisation

$$
\mathbf{y}_{0} \stackrel{\square_{1}(! \cdot e)}{\longrightarrow} \mathscr{T}_{0}^{\Lambda}\left(\mathbf{y}_{1}\right) \stackrel{\mathscr{T}_{0}^{\Lambda}(!)}{\longrightarrow} \mathscr{T}_{0}^{\Lambda}(1)
$$

where $\mathbf{y}_{1}(1)=\left\{\square_{1}\right\}$ and $! \cdot e$ is shorthand for $\mathscr{T}_{0}^{\Lambda}\left(!^{\mathbf{y}_{1}}\right)(e)$, with $!^{\mathbf{y}_{1}}: 0 \rightarrow \mathbf{y}_{1}$ the unique such morphism. In order for (20) to be generic, there should exist a lifting

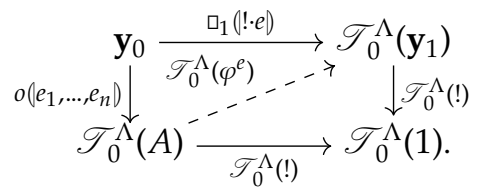

Now, the component at any $n$ of any $\operatorname{such} \varphi^{e}$ maps $o$ to some $\varphi_{n}^{e}(o) \in \mathbf{y}_{1}(n)=n$, and we have

$$
\begin{aligned}
\mathscr{T}_{0}^{\Lambda}\left(\varphi^{e}\right) \circ o\left(e_{1}, \ldots, e_{n}\right) & =\varphi^{e} \cdot\left(o\left(e_{1}, \ldots, e_{n}\right)\right) \\
& =\left(\varphi^{e} \cdot o\right)\left(\mid \varphi^{e} \cdot e_{1}, \ldots, \varphi^{e} \cdot e_{n}\right) \\
& =\square_{1}\left(\varphi^{e} \cdot e_{\varphi_{n}^{e}(o)}\right) .
\end{aligned}
$$

But because ! $e$ has no occurrence of any constant in $\mathbf{y}_{1}$, this entails that $e_{\varphi_{n}^{e}(o)}=! \cdot e_{\varphi_{n}^{e}(o)}^{\prime}$, for some $e_{\varphi_{n}^{e}(o)}^{\prime} \in \mathscr{T}_{0}^{\Lambda}(0)(0)$, and therefore

$$
\mathscr{T}_{0}^{\Lambda}\left(\varphi^{e}\right) \circ o\left(e_{1}, \ldots, e_{n}\right)=\square_{1}\left(! \cdot e_{\varphi_{n}^{e}(o)}^{\prime}\right)
$$

Finally, there are infinitely many factorisations of the original morphism which are the form $\mathscr{T}_{0}^{\Lambda}(!) \circ \square_{1}(! \cdot e)$, but only at most $n$ admitting a lifting $\varphi^{e}$, namely those $\square_{1}\left(! \cdot e^{\prime}\right)$ for which $e_{i}=! \cdot e^{\prime}$ for some $i \in n$. The given factorisation (20) thus cannot be generic.

Definition A.1. In any skew-monoidal category $\mathscr{C}$, for any monoid $X$, the category $X$-mod of (right) $X$-modules has as objects all $M \in \mathscr{C}$ equipped with an action $r: M \otimes X \rightarrow M$, making the following diagrams commute, where $e_{X}$ and $m_{X}$ are the unit and multiplication of $X$.

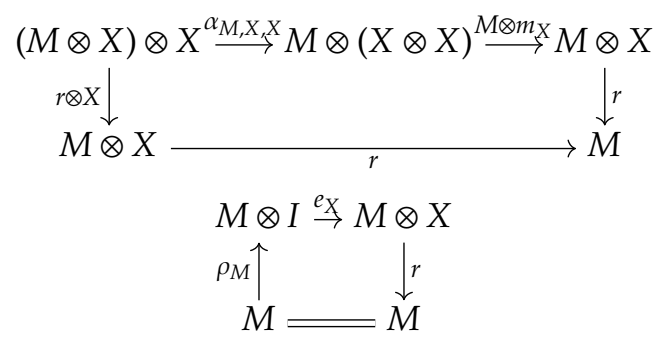

A module morphism is a morphism commuting with action.

Definition A.2. An action of a skew-monoidal category $(\mathscr{V}, \otimes, J)$ on a skew-monoidal category $(\mathscr{C}, \otimes, I)$ consists of a functor $\odot: \mathscr{C} \times \mathscr{V} \rightarrow \mathscr{C}$ equipped with natural transformations

$\rho_{A}: A \rightarrow A \odot J$ and $\alpha_{A, X, Y}: A \odot X \odot Y \rightarrow A \odot(X \otimes Y)$ such that the following diagrams commute.
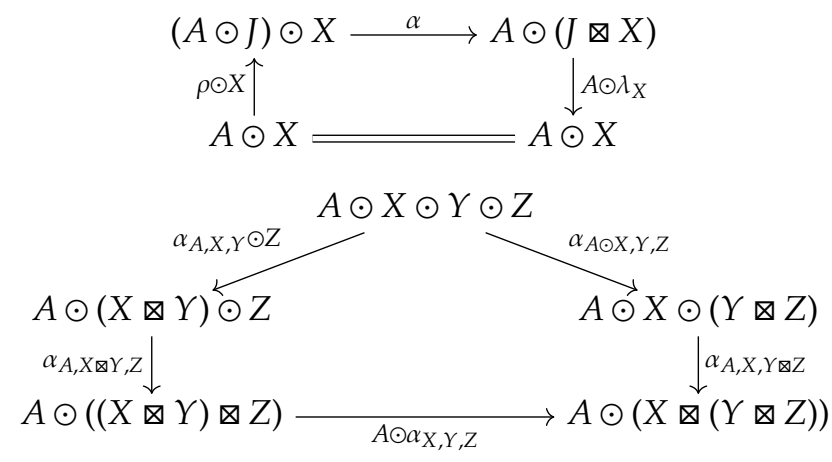

Definition A.3. A $\mathscr{V}$-strength on a functor $F: \mathscr{C} \rightarrow \mathscr{C}^{\prime}$ is a natural transformation $s_{A, X}: F(A) \odot X \rightarrow F(A \odot X)$ (where the first $\odot$ is the one of $\mathscr{C}^{\prime}$ and the second the one of $\mathscr{C}$ ), making the following diagrams commute.
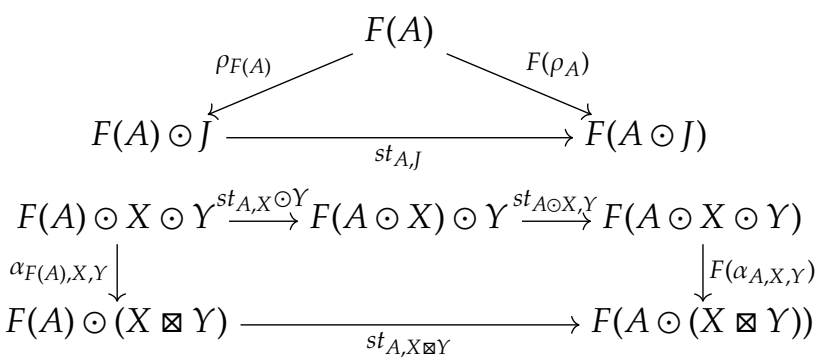

Given any skew-monoidal category $\mathscr{C}$, pointed $I$-modules form a skew monoidal category with tensor product $\otimes$, the original tensor product yielding an $I-\bmod _{I}$-action as in Remark ??. In fact, it further induces an $I-\bmod _{I}$-action on any power $\mathscr{C}^{n}$ of $\mathscr{C}$ by mapping any $\left(\left(A_{1}, \ldots, A_{n}\right), X\right)$ to $\left(A_{1} \otimes X, \ldots, A_{n} \otimes X\right)$.

Definition A.4. A structural strength on a functor $F: \mathscr{C}^{n} \rightarrow$ $\mathscr{C}$ is an $I-\bmod _{I}$-strength.

Lemma A.5. A natural transformation $F\left(A_{1}, \ldots, A_{n}\right) \otimes X \rightarrow$ $F\left(A_{1} \otimes X, \ldots, A_{n} \otimes X\right)$ forms a structural strength iff the following diagrams commute for all morphism $f: X \otimes Y \rightarrow U$ coequalising the parallel pair of (4). 


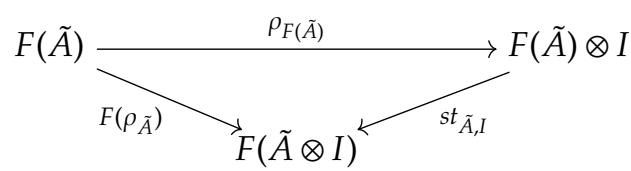

$(F(\tilde{A}) \otimes X) \otimes Y^{s t} \stackrel{\tilde{A}, X}{\longrightarrow} F(\tilde{A} \otimes X) \otimes Y^{s t} \stackrel{\tilde{\tilde{A} \otimes X} Y}{\longrightarrow} F((\tilde{A} \otimes X) \otimes Y)$

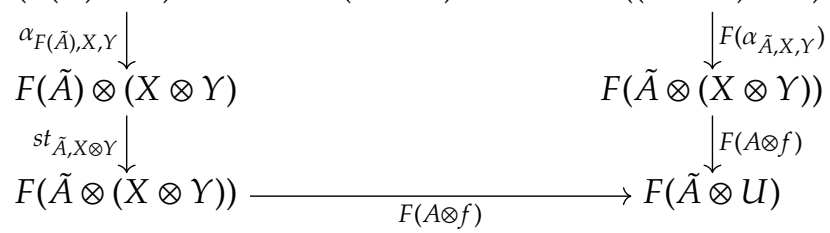

\section{B Proof of Theorem 2.15}

The theorem is proved in Coq (see supplementary material), but here is a perhaps more accessible explanation.

By [28, Theorem IV.1.2(ii)], a left adjoint is entirely determined by constructing the free $F$-monoid for each $X$. Monadicity then follows directly by Beck's theorem [28, Theorem VI.7.1(ii)]

Our main tool in this proof will be [16, Theorem 4.8]. In the notation of [16], the free $F$-monoid on $X$ is $\mu H(X)$, where $H(X, A)=G_{X}(A)$. We will rather denote it by $F^{\circledast}(X)$ or $A_{X}$ (where $H$ implicitly depends on $F$ ), and let $\varphi_{X}: H\left(X, A_{X}\right) \rightarrow$ $A_{X}$ denote the canonical isomorphism, whose components are denoted by

$$
\left[e_{X}, v_{X}, \sigma_{X}\right]: I+F\left(A_{X}\right)+X \otimes A_{X} \rightarrow A_{X} .
$$

The first step lies in proving that $A_{X}$ is indeed an $F$-monoid. The unit and $F$-algebra structure are clearly given by $e_{X}$ and $v_{X}$. Multiplication is more complicated, as it needs to be defined inductively.

Our first observation is:

Lemma B.1. For any $X, A_{X}$ is a pointed I-module.

Proof. The point is obviously given by

$$
I \stackrel{i n_{1}}{\longrightarrow} I+F\left(A_{X}\right)+X \otimes A_{X}=H\left(X, A_{X}\right) \stackrel{\varphi_{X}}{\longrightarrow} A_{X} .
$$

For the action of $I$, we apply [16, Theorem 4.8] to get a map $r: A_{X} \otimes I \rightarrow A_{X}$ making the following diagram commute,

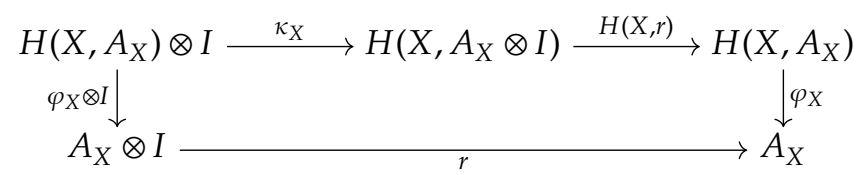

where $\kappa_{X}$ is defined up to commutation of coproduct with tensor by

$$
\begin{gathered}
I \otimes I+F\left(A_{X}\right) \otimes I+X \otimes A_{X} \otimes I \\
\downarrow \lambda_{I}+s t_{A_{X}, I}^{F}+\alpha_{X, A_{X}, I} \\
I+F\left(A_{X} \otimes I\right)+X \otimes\left(A_{X} \otimes I\right) .
\end{gathered}
$$

To show that $r$ is indeed a left inverse to $\rho$, we prove that both $i d$ and $r \circ \rho$ satisfy the defining equation of $\left(i d_{H\left(X, A_{X}\right)}\right)^{\#}$. The first does trivially, and the second because Diagram (21)
(Figure 1) commutes, where the upper part commutes by definition of skew monoidality and monoidal strength.

Let us now construct the multiplication of $A_{X}$. Letting $L(Y, B)=A_{X}+F(B)+Y \otimes B$, multiplication will follow as $s^{\#}$, where

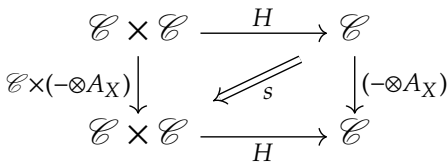

is defined by

$$
\begin{gathered}
H(Y, B) \otimes A_{X} \\
\mathbb{R} \\
I \otimes A_{X}+F(B) \otimes A_{X}+Y \otimes B \otimes A_{X} \\
\downarrow \lambda_{A_{X}}+s t_{B, A_{X}}^{F}+\alpha_{Y, B, A_{X}} \\
L\left(Y, B \otimes A_{X}\right) .
\end{gathered}
$$

We thus obtain by [16, Theorem 4.8] again a unique $m_{X}: A_{X} \otimes$ ${ }^{A_{X}} \rightarrow A_{X}$ making the Diagram (22) (Figure 1) commute. We now have our candidate $F$-monoid structure for $A_{X}$. Let us check that it satisfies the axioms, in successive lemmas.

Lemma B.2. The point $e_{X}$ is a left unit for $m_{X}$, i.e., the following diagram commutes.

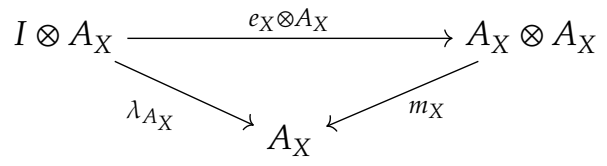

Proof. The composite $m_{X} \circ\left(e_{X} \otimes A_{X}\right)$ factors through $\varphi_{X} \otimes$ $A_{X}$, as

$$
\begin{aligned}
& I \otimes A_{X} \\
& \downarrow i n_{1} \otimes A_{X} \\
& \left(I+F\left(A_{X}\right)+X \otimes A_{X}\right) \otimes A_{X} \\
& \| \\
& H\left(X, A_{X}\right) \otimes A_{X} \stackrel{\varphi_{X} \otimes A_{X}}{\longrightarrow} A_{X} \otimes A_{X} .
\end{aligned}
$$

Diagram (23) (Figure 1) thus commute, whence the result.

Lemma B.3. The point $e_{X}$ is a right unit for $m_{X}$, i.e., the following diagram commutes.

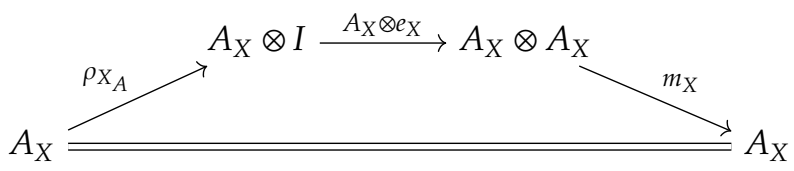

Proof. The identity on $A_{X}$ may be characterised as $\left(i d_{H\left(X, A_{X}\right)}\right)^{\#}$, so by uniqueness in[16, Theorem 4.8] it suffices to show that the top composite satisfies the same defining diagram. We do so separately for each term of $H\left(X, A_{X}\right)$. For the first term, the top morphism reduces to the identity, i.e., Diagram (24) (Figure 1) commutes. We use this to show that the defining diagram for the first term commutes: 


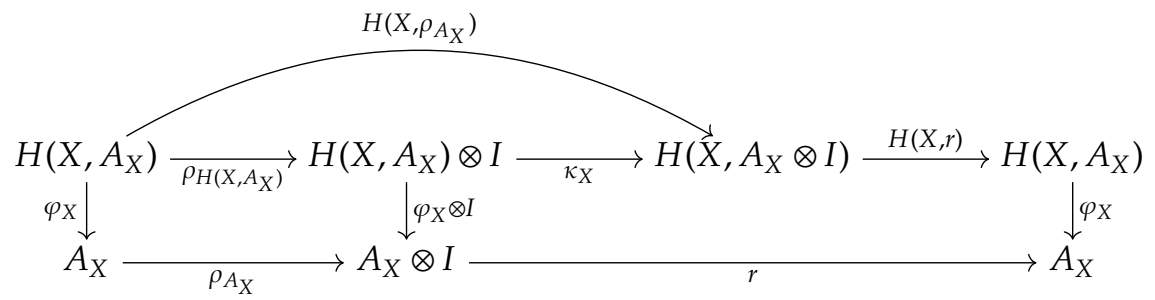

$(21)$

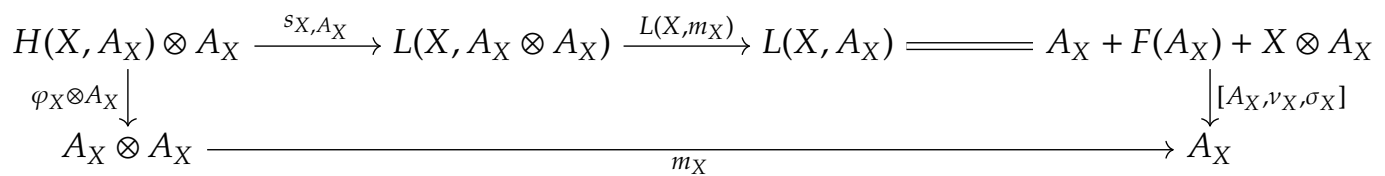

$(22)$

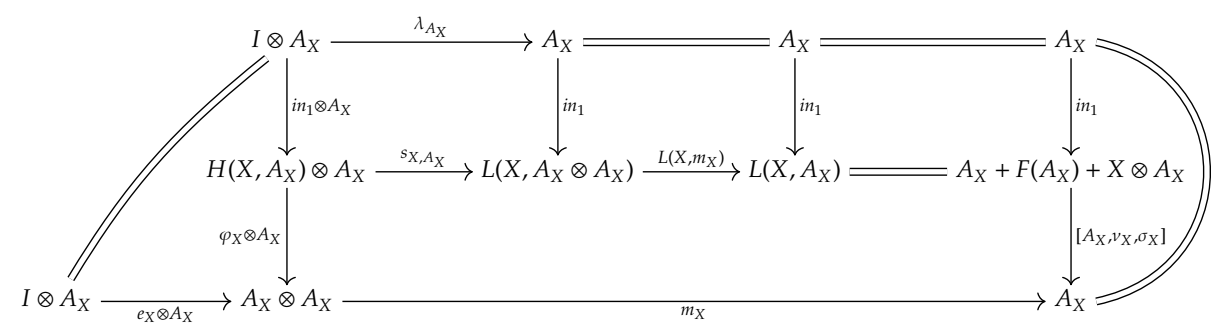

(23)

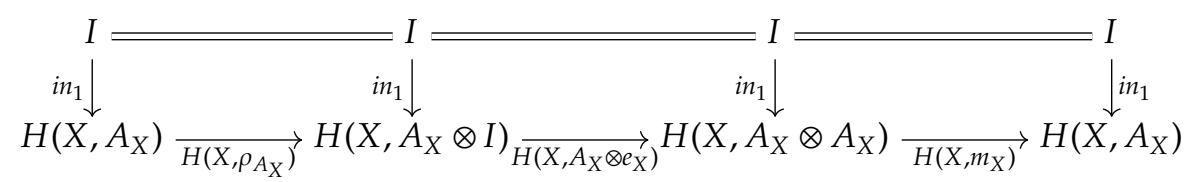

(24)

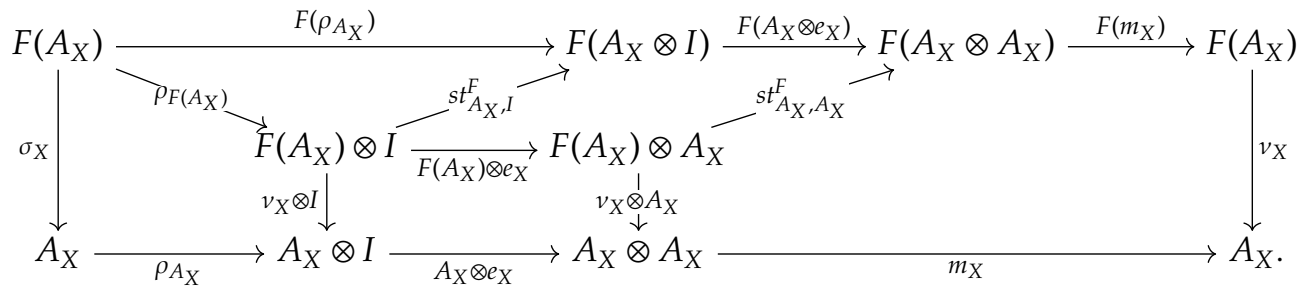

(25)

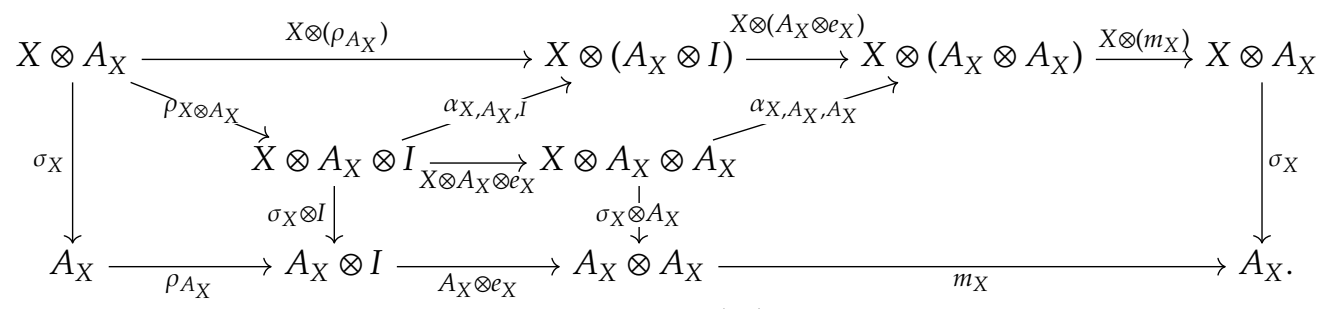

(26)

Figure 1. Diagrams for Appendix B

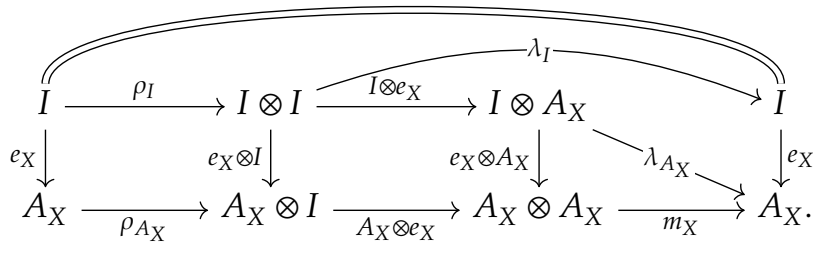

The triangle commutes by the previous lemma. For the second term, we obtain similarly that Diagram (25) (Figure 1) commutes. For the third term, we get Diagram (26), which commutes as desired. 
Lemma B.4. The multiplication $m_{X}$ is associative, i.e., the following diagram commutes.

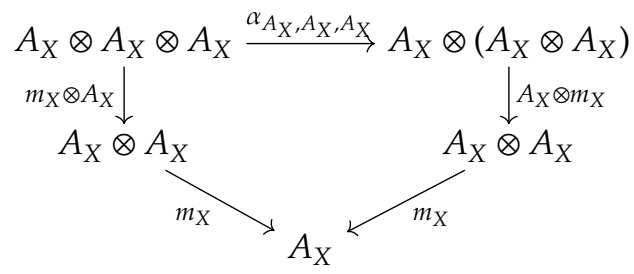

Proof. We apply [16, Theorem 4.8] again, with $J(B)=B \otimes$ $A_{X} \otimes A_{X}$, showing that both morphisms satisfy the defining diagram of $\left(s_{X, A_{X} \otimes A_{X}}^{\prime} \circ\left(s_{X, A_{X}} \otimes A_{X}\right)\right)^{\sharp}$, where the relevant natural transformation $s_{Y, B}^{\prime}$ is

$$
\begin{gathered}
A_{X} \otimes A_{X}+F\left(B \otimes A_{X}\right) \otimes A_{X}+Y \otimes\left(B \otimes A_{X}\right) \otimes A_{X} \\
\downarrow m_{X}, s t_{\left.B \otimes A_{X}, A_{X}, \alpha Y, B \otimes A_{X}, A_{X}\right]}^{F} \\
A_{X}+F\left(B \otimes A_{X} \otimes A_{X}\right)+Y \otimes\left(B \otimes A_{X} \otimes A_{X}\right) .
\end{gathered}
$$

Let us start with the first component of the first morphism, which commutes easily:

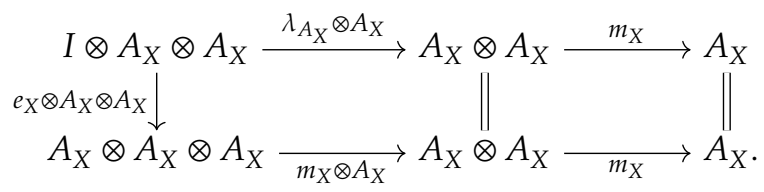

The first term of the second morphism is a bit more complex: see Diagram (27) (Figure 2). Both second terms are treated as Diagrams (28) and (29). Commutation of (29) is subtler than it looks, because the top pentagon only commutes upon postcomposition with $F\left(A_{X} \otimes m_{X}\right)$, using Lemma A.5. But in order for this lemma to apply, we should show that the following diagram commutes,

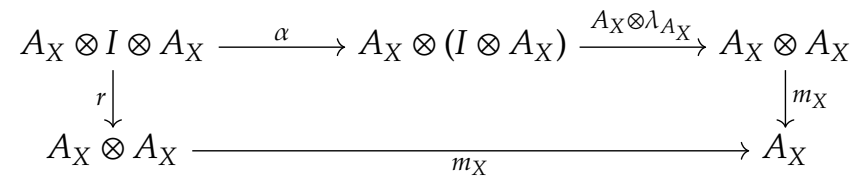

which holds by...

Finally, both third terms are displayed as Diagrams (30) and (31).

Lemma B.5. The F-algebra and monoid structure on $A_{X}$ are compatible, i.e., the following diagram commutes.

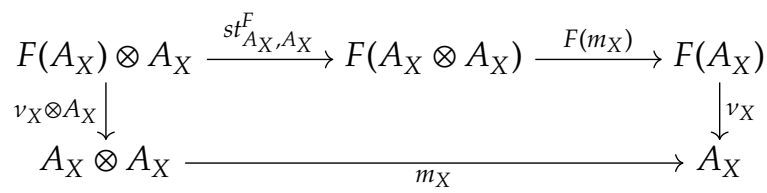

Proof. This is merely the second term of the defining diagram for $m_{X}$.

We have shown that $A_{X}$ is indeed and F-monoid. It remains to prove its initiality property.
Lemma B.6. $A_{X}$ is the initial F-monoid over $X$.

Proof. Let $M$ denote any $F$-monoid, with structure

$$
\left[e_{M}, v_{M}, m_{M}\right]: I+F(M)+M \otimes M \rightarrow M,
$$

and let $f: X \rightarrow M$ denote any morphism. We let the desired extension $\bar{f}$ arise as $i d_{H\left(X, A_{X}\right)}^{\sharp}$, using the fact that $M$ admits an $H$-algebra structure $\varphi_{M}$ defined as the composite

$$
\begin{gathered}
H(X, M)=I+F(M)+X \otimes M \\
\downarrow I+F(M)+f \otimes M \\
I+F(M)+M \otimes M \\
\downarrow\left[e_{M}, v_{M}, m_{M}\right] \\
M,
\end{gathered}
$$

i.e., $\bar{f}$ is the unique morphism making the following diagram commute.

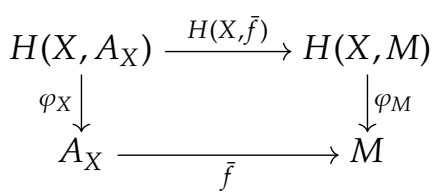

We need to show that it is a morphism of $F$-monoids, that precomposing it with $\eta_{X}: X \rightarrow A_{X}$ yields $f$, and that it is the unique such. The first point is a direct consequence of $\bar{f}$ being an $H(X,-)$-algebra morphism, which holds by construction. For the second point, it holds because

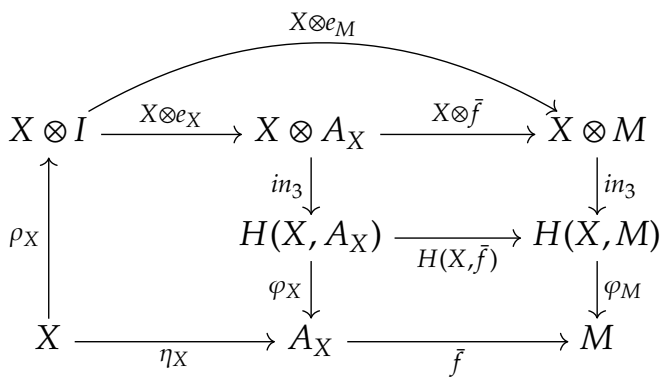

commutes, which in turn holds because

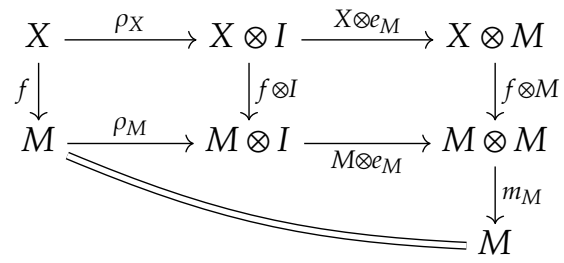

commutes. Finally, let us show that any morphism fulfilling the relevant conditions would in particular be a morphism of $H(X,-)$-algebras, hence equal to $\bar{f}$, as desired. For this, assume given an $F$-monoid morphism $f^{\prime}: A_{X} \rightarrow M$ such that $f^{\prime} \circ \eta_{X}=f$. We show that $f^{\prime}$ is a morphism of $H(X,-)$ algebras, term by term. For the first and second terms, it follows directly from $f^{\prime}$ being a morphism of $F$-monoids. For the third term, this follows from commutation of 


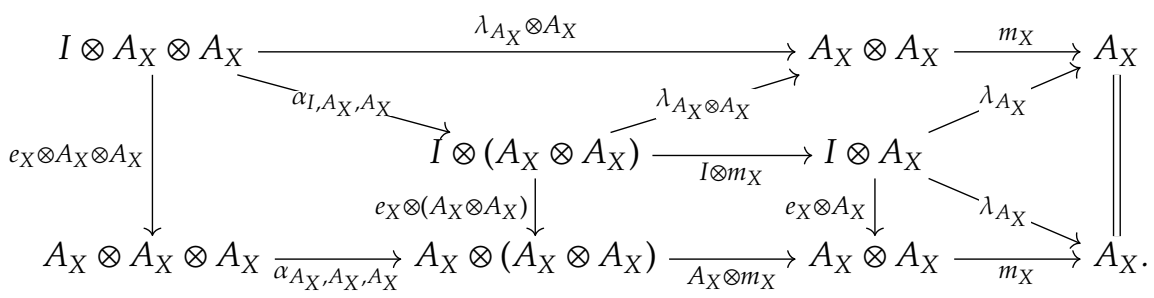

(27)

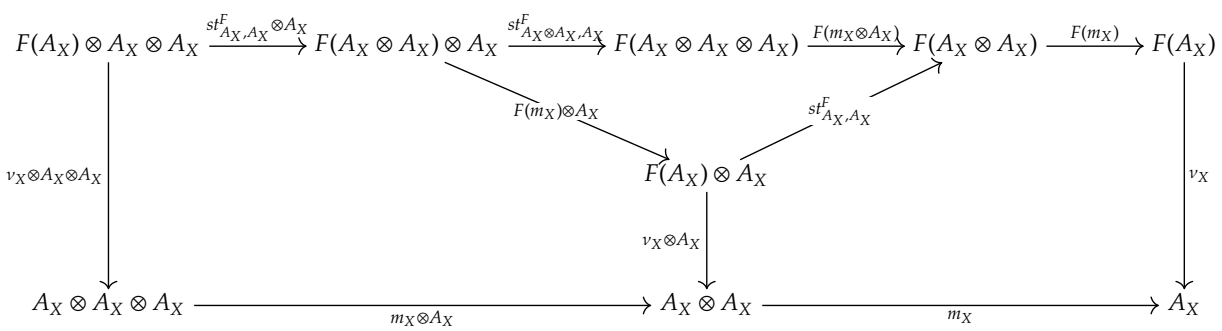

(28)

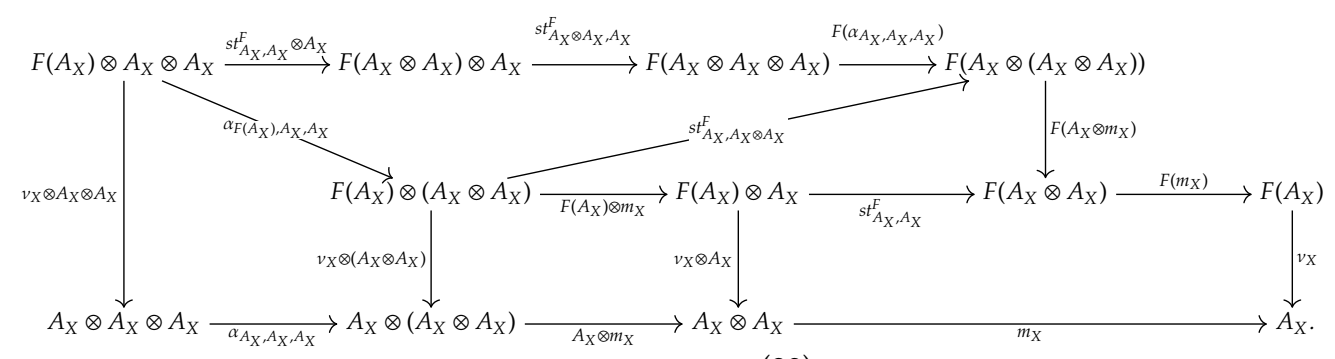

(29)

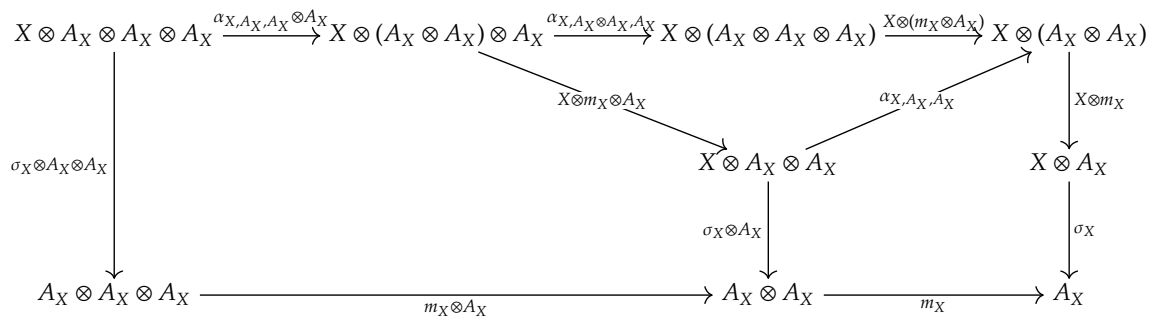

(30)

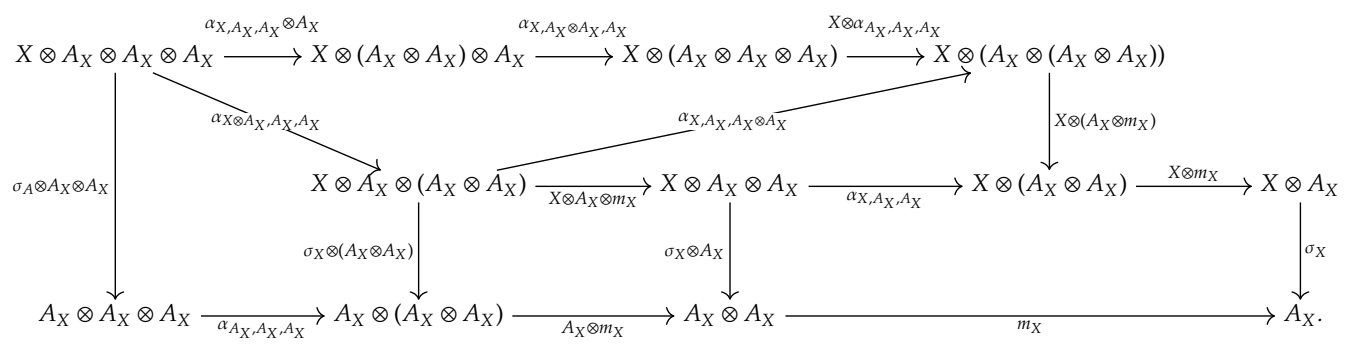

$(31)$

Figure 2. Diagrams for the proof of Lemma B.4

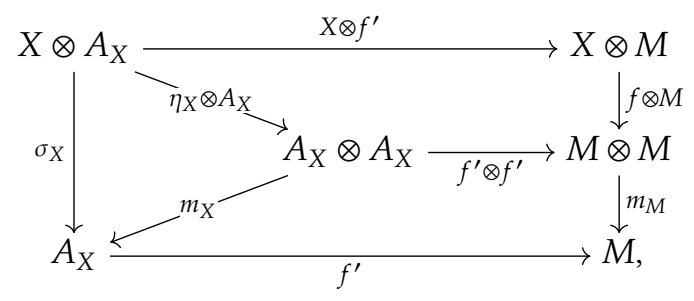

where the triangle commutes because the diagram of Figure 3 does. 


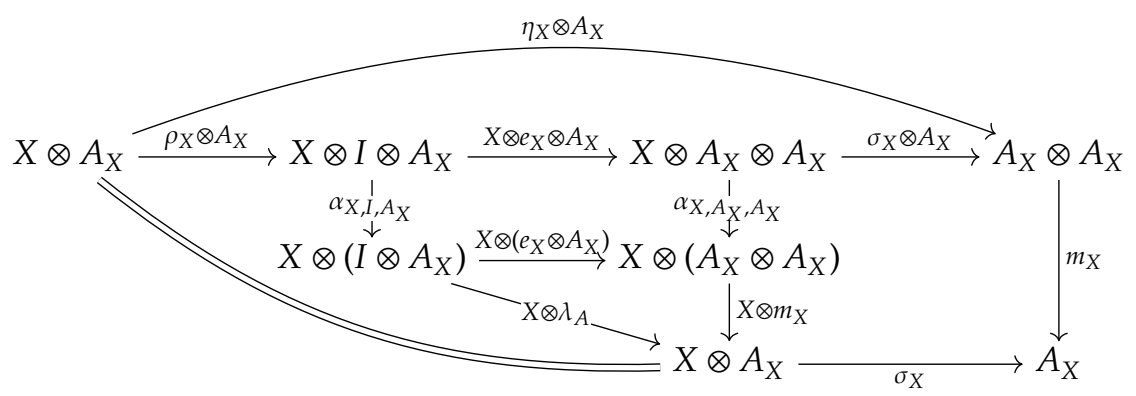

Figure 3. Diagram for Lemma B.6

\section{Proof of Theorem 2.19}

For familiality of $F^{\circledast}$, let us start with the following observations:

- $I, F$, and $\otimes$ are all familial, and familial functors are well-known to be stable under coproducts and composition, so each $G_{-}^{n}(0)$ is familial;

- $0=G_{X}^{0}(0) \rightarrow G_{X}^{1}(0)$ is trivially cartesian, so by induction each $\partial_{X}^{n}$ is cartesian by familiality of $F$ and $\otimes$

- by a similar argument, each $\partial_{X}^{n}$ is monic.

By generalising the pullback lemma, pullback squares are stable under $\omega$-composition in locally finitely presentable categories, so each $\omega$-composite

$$
\lambda_{X}^{n}=\left(\ldots \circ \partial_{X}^{n+1} \circ \partial_{X}^{n}\right): G_{X}^{n}(0) \rightarrow F^{\circledast}(X),
$$

is cartesian hence preserves generics by Proposition 2.17. Similarly, each $\lambda_{X}^{n}$ is monic by [3, Proposition 1.62]. Thus the generic factorisation of any $\mathbf{y}_{c} \rightarrow F^{\circledast}(X)$ is given by first factoring through some $G_{X}^{n}(0)$ by finite presentability of $\mathbf{y}_{c}$, then finding some $G_{-}^{n}(0)$-generic factorisation by familiality of $G_{-}^{n}(0)$, and then conclude by cartesianness of $G_{-}^{n}(0) \rightarrow F^{\circledast}$. We finally conclude that $F^{\circledast}$ is familial by [18, Lemma 3.9(ii)].

Let us now show that $\eta^{F}$ is monic and cartesian. We take the same notation as in the proof of Theorem 2.15: $A_{X}$ denote $F^{\circledast}(X)$, with $\varphi_{X}: H_{F}\left(X, A_{X}\right) \stackrel{\sim}{\rightarrow} A_{X}$ the structural isomorphism, which decomposes as $\left[e_{X}, v_{X}, \sigma_{X}\right]: I+F\left(A_{X}\right)+$ $X \otimes A_{X} \rightarrow A_{X}$.

The component of $\eta^{F}$ at $X$ is then the composite

$$
\begin{gathered}
F(X) \\
\downarrow F\left(\rho_{X}\right) \\
F(X \otimes I) \\
\downarrow i_{2}\left(X \otimes i n_{1}\right) \\
G_{X}\left(X \otimes G_{X}(0)\right) \\
\downarrow G_{X}\left(i n_{3}\right) \\
G_{X}\left(G_{X}\left(G_{X}(0)\right)\right) \\
\downarrow \lambda_{X}^{3} \\
A_{X},
\end{gathered}
$$

of which we have seen that the last factor is cartesian and monic. But $\rho_{X}$ and coproduct injections are cartesian and monic, so by preservation of pullbacks the composite is again cartesian and monic, as desired.

\section{Howe contexts}

This section collects proofs and intermediate results for $\S 4$.

\section{D.1 Basic properties}

Lemma D.1. Any morphism $f: P^{\prime} \rightarrow L$ with $P^{\prime}$ some state type, and $L$ some transition type with associated copairing $[s, t]: P+Q \rightarrow L$, lifts through $[s, t]$ in the sense that there exists $k$ making the following diagram commute.

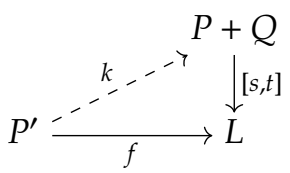

Proof. This follows directly from the fact that $\mathscr{D}[s, t]$ is an isomorphism.

As presheaf categories, $\mathscr{C}_{0}$ and $\mathscr{C}$ are toposes, hence in particular regular, i.e., they admit strong epi - mono factorisations, and strong epis are stable under pullbacks. Our assumptions furthermore entail that such image factorisations are preserved by tensor product on the left.

Lemma D.2. Each functor $-\otimes C$ preserves image (= strong epi/mono) factorisations.

Proof. By preservation of colimits, hence of strong epis, and connected limits, hence of monos.

Lemma D.3. For any $X \in \mathscr{C}$, we have $\mathscr{D}\left(\varepsilon_{X}\right)=i d_{\mathscr{D}(X)}$.

Proof. $\mathscr{D}\left(\varepsilon_{X}\right)$ becomes the identity upon composition with the unit $\eta_{\mathscr{D}(X)}$, which is itself an identity by hypothesis.

\section{D.2 Substitution-closed bisimulation}

Proof of Lemma 4.6. There is an abstract-nonsense proof here, but let us be concrete to aid intuition. First of all, $U_{0}$ being monadic is automatically an iso-fibration. For a choice of the pullback $\mathscr{D}^{*}(\mathscr{E})$, we take the standard construction as a subcategory of the product.

Let us first consider the left adjoint $\mathscr{E} \uparrow \mathscr{M}$ to $\mathscr{D} \uparrow \mathscr{E}$. For any $E \in \mathscr{E}$, we have by coreflection $\eta_{U_{0}(E)}: U_{0}(E) \stackrel{\sim}{\rightarrow}$ 
$\mathscr{D}\left(\mathscr{M}\left(U_{0}(E)\right)\right)$, so by lifting along this isomorphism we find an isomorphism $E \uparrow \eta_{U_{0}(E)}: E \rightarrow E^{\prime} U_{0}\left(E^{\prime}\right)=\mathscr{D}\left(\mathscr{C}\left(U_{0}(E)\right)\right)$ and $U_{0}\left(E\left\lceil\eta_{U_{0}(E)}\right)=\eta_{U_{0}(E)}\right.$. By making a global choice of such $E^{\prime}$, we may let $\mathscr{E} \uparrow \mathscr{M}(E)=\left(\mathscr{M}\left(U_{0}(E)\right), E^{\prime}\right)$, which is in the pullback $\mathscr{D}^{*}(\mathscr{E})$ by construction. This assignment extends straightforwardly to a functor by adjusting any $E \rightarrow F$ to $E^{\prime} \cong E \rightarrow F \cong F^{\prime}$. Let us now prove this functor is left adjoint to $\mathscr{D} \uparrow \mathscr{E}$. Indeed, $\mathscr{D} \uparrow \mathscr{E}\left(\mathscr{M}\left(U_{0}(E)\right), E^{\prime}\right)=E^{\prime}$, so it makes sense to take the unit to be the given isomorphism $E \cong E^{\prime}$. This is natural by construction of the above adjustment. Furthermore, for any $f: E \rightarrow(\mathscr{D} \uparrow \mathscr{E})(B)$ in $\mathscr{E}$ with $B=\left(B_{1}, B_{2}\right)$, the pair $\left(\widetilde{U_{0}(f)}, f \circ\left(E \uparrow \eta_{U_{0}(E)}\right)^{-1}\right): \mathscr{E} \uparrow$ $\mathscr{M}(E) \rightarrow B$, where $\widetilde{U_{0}(f)}: \mathscr{M} U_{0} E \rightarrow B_{1}$ denotes the transpose of $U_{0}(f): U_{0}(E) \rightarrow U_{0}(\mathscr{D} \uparrow \mathscr{E}(B))=\mathscr{D}\left(\mathscr{D}^{*}\left(U_{0}\right)(B)\right)=$ $\mathscr{D}\left(B_{1}\right)$, and is indeed in $\mathscr{D}^{*}(\mathscr{E})$ by the triangle identities. It furthermore verifies the expected identity, with candidate unit given at $E$ by $E \uparrow \eta_{U_{0}(E)}$ itself: $\mathscr{D} \uparrow \mathscr{E}\left(\widetilde{U_{0}(f)}\right), f \circ(E \uparrow$ $\left.\left.\eta_{U_{0}(E)}\right)^{-1}\right) \circ \eta=f \circ\left(E \uparrow \eta_{U_{0}(E)}\right)^{-1} \circ\left(E \uparrow \eta_{U_{0}(E)}\right)=f$, and does so uniquely because any other candidate must have the form $\left(g, f^{\prime}\right)$ with $g: \mathscr{M} U_{0} E \rightarrow B_{1}$ and $\mathscr{D}(g)=U_{0}\left(f^{\prime}\right)$ hence $g=\widetilde{U_{0}(f)}$ by uniqueness in the universal property.

Finally, briefly, $\mathscr{L}$ is given on any object $C \in \mathscr{C}$ by a choice of opcartesian lifting of $C$ along $\mathscr{M}\left(\eta_{\mathscr{D} C}^{0}\right): \mathscr{M} \mathscr{D C} \rightarrow$ $\mathscr{M} T_{0} \mathscr{D C}$, where $T_{0}$ denotes the monad induced by $U_{0}$, the unit being given by the chosen lifting, so that in particular $\mathscr{D}\left(\eta_{C}\right)=\eta_{\mathscr{D C}}^{0}$.

Because $\mathscr{D}(X)$-modules are algebras for the monad $-\otimes X$, which preserves all colimits, we have

Lemma D.4. The forgetful functor $\mathscr{F}: \mathscr{D}(X)-\bmod \rightarrow \mathscr{C}_{0}$ is monadic, and creates all limits, colimits, and image factorisations.

Proof. The result holds generally for limits, and for colimits follows from their preservation. Finally, consider any image factorisation $M \stackrel{e}{\rightrightarrows} N \stackrel{m}{\longrightarrow} P$ of some given X-module morphism. Then because tensoring with $X$ on the right preserves image factorisations, $M \otimes X \stackrel{e \otimes X}{\longrightarrow} N \otimes X \stackrel{m \otimes X}{\longrightarrow}$ $P \otimes X$ is a again an image factorisation, so we get a lifting

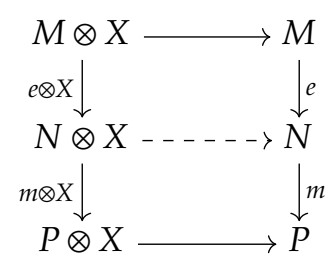

which equips $N$ with $X$-module structure.

Lemma D.5. In a pullback

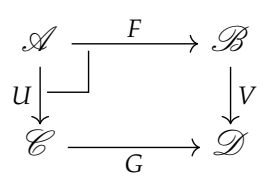

of functors such that $G$ preserves and $V$ creates a given type of limit or colimit, then $F$ preserves and $U$ creates them.

Proof. Let us treat the case of colimits, the one for limits being symmetric. Consider any functor $A: \mathscr{J} \rightarrow \mathscr{A}$ and colimiting cocone $\sigma^{\mathscr{C}}$ for $U A$. By preservation of colimits for $A$, $\sigma^{\mathscr{C}}$ is mapped to a colimiting cocone $\sigma^{\mathscr{D}}$ for $G U A=V F A$ by $G$, which by creation of colimits for $A$ lifts uniquely to a cocone $\sigma^{\mathscr{B}}$ for $F A$, which is colimiting. The colimiting cocone formed by componentwise pairing of $\sigma^{\mathscr{C}}$ and $\sigma^{\mathscr{B}}$ thus yields a colimiting cocone $\sigma^{\mathscr{A}}$ from $A$ over $\sigma^{\mathscr{C}}$. It remains to prove that this is the unique cocone over $\sigma^{\mathscr{C}}$. But any cocone $\tau$ over $\sigma^{\mathscr{C}}$ maps to some cocone $F(\tau)$ over $\sigma^{\mathscr{D}}$, which by creation of colimits for $V$ has to be precisely $\sigma^{\mathscr{B}}$, hence $\tau=\sigma^{\mathscr{A}}$ by universal property of pullback.

Corollary D.6. The forgetful functor $\mathscr{F}: \mathscr{D}(X)-\bmod \rightarrow$ $\mathscr{C}_{0}$ creates unions.

Proof. Consider any family of relations $R_{i} \hookrightarrow M \times N$ in $\mathscr{D}(X)$-mod, their union in $\mathscr{C}_{0}$ is given by the image of the copairing $\sum_{i} R_{i} \rightarrow M \times N$. By creation of colimits the copairing lifts to a copairing in $\mathscr{D}(X)$-mod, and the result follows by creation of image factorisations.

Thus, because $\mathscr{D}$, as a left and right adjoint, preserves all limits and colimits, we get

Corollary D.7. The forgetful functor $\mathscr{F}^{\prime}: X-\operatorname{Mod} \rightarrow \mathscr{C}_{0}$ creates all limits and colimits.

Finally, we proceed similarly for image factorisations.

Lemma D.8. In the situation of Lemma D.5, if $G$ preserves and $V$ creates image factorisations, then $F$ preserves and $U$ creates them.

Remark D.9. It may not be entirely clear what is meant by creation of image factorisations: we mean that for all $f: b_{1} \rightarrow b_{2}$ and image factorisation $m \circ e=V(f)$, there is a unique factorisation $m^{\prime} \circ e^{\prime}$ of $f$ such that $V\left(e^{\prime}\right)=e$ and $V(m)=m^{\prime}$, which is furthermore an image factorisation (i.e., $e^{\prime}$ is a strong epi and $m^{\prime}$ is a mono).

Proof. Similar to the proof of Lemma D.5, using the fact, obvious for limits and colimits but perhaps less so for image factorisations, that the canonical embedding $\mathscr{A} \rightarrow \mathscr{C} \times \mathscr{B}$ reflects image factorisations.

Corollary D.10. The forgetful functor $\mathscr{F}^{\prime}: X-\operatorname{Mod} \rightarrow \mathscr{C}$ creates image factorisations.

Proof of Proposition 4.10. Same idea as Corollary D.6 using the previous lemmas. 


\section{D.3 Signatures, models, and initiality}

Lemma D.11. The forgetful functor $\check{\Sigma}_{1}-\mathrm{alg}_{v} \rightarrow \Sigma_{0}$-mon is a fibration.

Proof. By [4, Lemma 6.9].

Lemma D.12. The forgetful functor $\mathscr{W}: \check{\Sigma}_{1}^{\Lambda}-\operatorname{alg}_{v} \rightarrow \Sigma_{0}^{\Lambda}$-Mon is a morphism of fibrations, i.e., it preserves cartesian morphisms.

Proof of Theorem 4.20. Consider any transition $\Sigma_{0}^{\Lambda}$-monoid $X$. Assume that $\mathscr{L}(X)$ is free over $X$ in the fibre over $\mathscr{D}^{\prime}(X)$. We show that $\mathscr{L}(X)$ is free over $X$ in the whole category. For this, consider any $f: X \rightarrow \mathscr{W}(Y)$. By cartesian lifting of $Y$ along $\mathscr{D}^{\prime}(f)$, we obtain a cartesian morphism $f^{\prime}: Y^{\prime} \rightarrow$ $Y$ in $\check{\Sigma}_{1}^{\Lambda}-\operatorname{alg}_{v}$. By the lemma, $\mathscr{W}\left(f^{\prime}\right)$ is cartesian, which yields a unique vertical $g: X \rightarrow \mathscr{W}\left(Y^{\prime}\right)$, such that $\mathscr{W}\left(f^{\prime}\right) \circ g=f$. By initiality of $\mathscr{L}(X)$, we thus obtain a unique morphism $\tilde{g}: \mathscr{L}(X) \rightarrow Y^{\prime}$ of vertical algebras such that $\mathscr{W}(\tilde{g}) \circ \eta_{X}=g$. The composite $f^{\prime} \circ \tilde{g}$ is thus such that $\mathscr{W}\left(f^{\prime} \circ \tilde{g}\right) \circ \eta_{X}=$ $\mathscr{W}\left(f^{\prime}\right) \circ g=f$, as desired. Finally, given any $\tilde{f}: \mathscr{L}(X) \rightarrow$ $Y$ satisfying this, by cartesianness of $f^{\prime}$ we find a unique vertical $h: \mathscr{L}(X) \rightarrow Y^{\prime}$ such that $f^{\prime} \circ h=\tilde{f}$. But then $g^{\prime}=\mathscr{W}(h) \circ \eta_{X}$ is such that $\mathscr{W}\left(f^{\prime}\right) \circ g^{\prime}=f$, hence $g^{\prime}=g$ by uniqueness in the universal property of $f^{\prime}$. By uniqueness in the universal property of $\mathscr{L}(X)$, we thus obtain $h=\tilde{g}$ and so $\tilde{f}=f^{\prime} \circ \tilde{g}$ as desired.

We have thus reduced the goal to proving existence of free algebras in each fibre of $\mathscr{D}^{\prime}$. By $[34, \S 2$.Theorem] and vertical finitarity the result boils down to cocompleteness of each fibre $\Sigma_{0}-$ Mon $_{X}$ over any $X \in \Sigma_{0}^{\Lambda}$-mon. But $\Sigma_{0}-$ Mon $_{X}$ is equivalent to the full subcategory $\mathscr{M}^{\prime}(X) / p v \Sigma_{0}$-Mon of the coslice category $\mathscr{M}^{\prime}(X) / \Sigma_{0}$-Mon spanning pseudo-vertical morphisms (notably thanks to the cancellation properties of pseudo-vertical morphisms), so it suffices to prove that the latter is cocomplete. Finally, as the coslice of a cocomplete category, $\mathscr{U}^{\prime}(X) / \Sigma_{0}$-Mon is cocomplete, and pseudovertical morphisms are closed under colimits therein, hence the result.

Let us now consider monadicity, first of vertical algebras over transition $\Sigma_{0}$-monoids. For this, we apply Beck's theorem: consider any parallel pair $f, g: X \rightarrow Y$ in $\Sigma_{0}$-Mon where $X$ and $Y$ are equipped with vertical $\breve{\Sigma_{1}}$-algebra structure, $(f, g)$ is a parallel pair in $\check{\Sigma}_{1}-\operatorname{alg}_{v}$, which admits an absolute coequaliser, say $q: Y \rightarrow Z$, in $\Sigma_{0}$-Mon. By absoluteness, $\check{\Sigma}_{1}(q)$ is a coequaliser of $\left(\check{\Sigma}_{1}(f), \check{\Sigma}_{1}(q)\right)$, so we get a unique map $z$ as below.

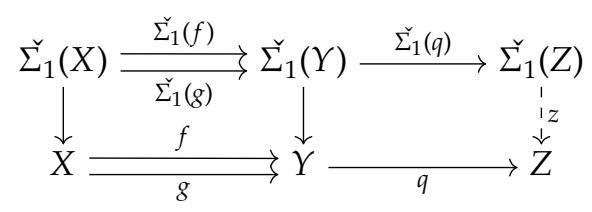

Let us show that $z$ is vertical: because $Y$ is vertical, the righthand square is mapped by $\mathscr{D}^{\prime}$ to

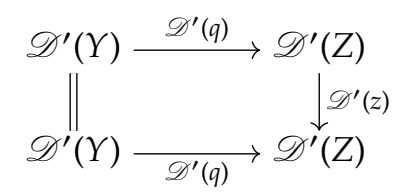

and, $q$ being absolute, $\mathscr{D}^{\prime}(q)$ is a coequaliser, hence epi, so $\mathscr{D}^{\prime}(z)=i d$, as desired.

By absoluteness of $q$, this is clearly the unique lifting of $q$ to vertical algebras, and furthermore it makes $(Z, z)$ a coequaliser in vertical algebras, which shows that the forgetful functor creates the needed coequalisers for Beck's theorem to apply.

\section{D.4 Weak compositionality}

Proof of Proposition 4.23. The right-hand morphism in (13) factors as $\Sigma_{1}(\mathbf{Z}) \stackrel{\gamma_{\mathbf{Z}}}{\longrightarrow} \check{\Sigma}_{1}(\mathbf{Z}) \rightarrow \mathbf{Z}$, where the second factor is the structure map of $\mathbf{Z}$, which is an isomorphism by Lambek's lemma, so we find a first lifting $r^{\prime}: L \rightarrow \check{\Sigma}_{1}(\mathbf{Z})$. Then, the opcartesian lifting of a pointwise surjective morphism is again pointwise surjective by construction, the $\operatorname{map} \gamma_{\mathbf{Z}}: \Sigma_{1}(\mathbf{Z}) \rightarrow$ $\check{\Sigma}_{1}(Z)$ is pointwise surjective, so we find a transition $k: L \rightarrow$ $\Sigma_{1}(Z)$ such that $\gamma_{\mathbf{Z}} \circ k=r^{\prime}$. It remains to prove that the upper triangle commutes. It certainly would commute by uniqueness if we had $e=k \backslash s$. But we have seen that $\mathscr{D}(\mathscr{U}(\mathbf{Z}))=0^{\circledast}$, so the $\Sigma_{0}$-monoid structure of $0^{\circledast}$ yields an isomorphism $\varphi^{0}: \Sigma_{0}(\mathscr{D}(\mathscr{U}(\mathbf{Z}))) \rightarrow \mathscr{D}(\mathscr{U}(\mathbf{Z}))$. Furthermore, the diagram in Figure 4 commutes, the middle square by construction of $\gamma_{\mathbf{Z}}$. We thus have

$$
\varepsilon_{\mathscr{U}(\mathbf{Z})} \circ \mathscr{M}\left(\varphi^{0}\right) \circ e=r \circ s=\varepsilon_{\mathscr{U}(\mathbf{Z})} \circ \mathscr{M}\left(\varphi^{0}\right) \circ(k \backslash s) .
$$

By verticality, we obtain $e=k \backslash s$, as desired.

\section{E Howe's method}

\section{E.1 Prebisimulations}

In this section, we provide some detail on how to adapt [21, $\S 5.2]$ to the present setting.

Lemma E.1. The set of copairings $[s, t]: P+Q \rightarrow L$ cofibrantly generates a strong factorisation system on $\mathscr{C}, \operatorname{say}(\mathscr{V}, \mathscr{H})$.

Proof. The construction follows the small object argument for strong factorisation systems, which works in all locally presentable categories [8].

Lemma E.2. A morphism $f$ is in $\mathscr{V}$ iff $\mathscr{D}(f)$ is an isomorphism; it is in $\mathscr{H}$ iff it is $\mathscr{D}$-cartesian.

Proof. By construction, all maps in $\mathscr{V}$ are transfinite composites of pushouts of copairings $[s, t]$. But the latter are mapped to isomorphisms by $\mathscr{D}$ by $(\mathrm{H} 3)$, hence so are all maps in $\mathscr{V}$ because isomorphisms are stable under pushouts and transfinite composition. Conversely, let $f: X \rightarrow Y$ be such that $\mathscr{D}(f)$ is an isomorphism. We thus have a commuting square 


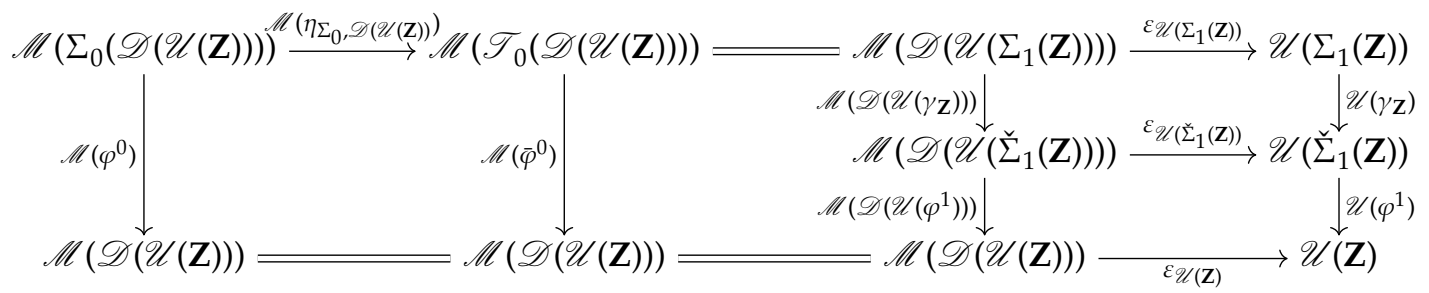

Figure 4. Diagram for the proof of Proposition 4.23

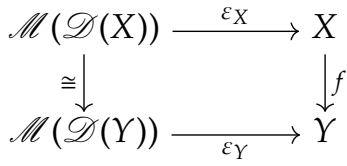

with both horizontal maps in $\mathscr{V}$, which entails $f \in \mathscr{V}$ by right cancellation.

We call morphisms in $\mathscr{V}$ pseudo-vertical.

Corollary E.3. The factorisations of Corollary 4.5 are particular cases.

Lemma E.4. Any morphism $f: C \rightarrow \mathscr{M}(A)$ is in the essential image of $\mathscr{M}$.

Proof. Because $\mathscr{M}(A)$ is empty on transition types, so is $C$.

Lemma E.5. Given any prebisimulation $m: R \hookrightarrow \mathscr{D}(X) \times$ $\mathscr{D}(Y) \cong \mathscr{D}(X \times Y)$, the cartesian factor $\bar{m}: \bar{R} \rightarrow X \times Y$ of the transpose $\check{m}: \mathscr{M}(R) \rightarrow X \times Y$ is a bisimulation.

Proof. Just as [21, Proposition 5.8], up to the following two glitches. First, the transpose $\check{m}$ is not obviously monic. But consider any parallel pair of arrows $f, g: A \rightrightarrows \mathscr{M}(R)$. By Lemma E.4, $f$ and $g$ are in the image of $\mathscr{M}$, say as $f=$ $\mathscr{M}\left(f^{\prime}\right)$ and $g=\mathscr{M}\left(g^{\prime}\right)$, so by transposition, we have that $m$ coequalises $f^{\prime}$ and $g^{\prime}$. But $m$ is monic by hypothesis, so $f^{\prime}=g^{\prime}$, whence $g=f$, as desired. The second glitch is that $\bar{m}$ is not obviously monic either. But by density, it is enough to show that it is monic on parallel morphisms $f, g: A \rightarrow$ $\bar{R}$, for $A$ some basic object. So consider any such $f$ and $g$ such that $\bar{m} \circ f=\bar{m} \circ g$. If $A=P$, then by Lemma D.1, we obtain liftings $f^{\prime}$ and $g^{\prime}$ of $f$ and $g$ through the first factor $l: R \rightarrow \bar{R}$. Because the composite $\bar{m} \circ l=\check{m}$ is monic we have $f^{\prime}=g^{\prime}$, hence $f=l \circ f^{\prime}=l \circ g^{\prime}=g$, as desired. If finally, $A=L$ appears in some transition type $P \stackrel{s}{\rightarrow} L \stackrel{t}{\leftarrow} Q$, then by the previous case $f \circ s=g \circ s$ and $f \circ t=g \circ t$, and so $f$ and $g$ are both diagonal liftings for the following square

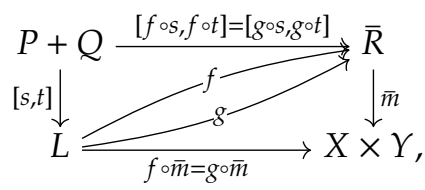

hence are equal by uniqueness of lifting in strong factorisation systems.

Because the image under $\mathscr{D}$ of any bisimulation $R \rightarrow X^{2}$ is a prebisimulation, we have

Corollary E.6. Calling PreBisim $(X)$ andBisim $(X)$ the posets of prebisimulations and bisimulations over $\mathrm{X}^{2}$, the monotone

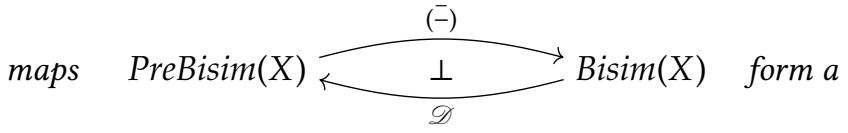

Galois connection.

Furthermore, we also have a natural notion of prebisimilarity:

Proposition E.7. Prebisimulations are stable under union.

Definition E.8. The union of all prebisimulations over any fixed object is called prebisimilarity and denoted by $\sim_{X}^{\vee}$.

In fact, this all lifts to substitution-closed prebisimulations:

Definition E.9. An $X$-prebisimulation is a relation $R \hookrightarrow$ $M^{2}$ in $X-\bmod$ such that $\mathscr{U}_{0}(R)$ is a prebisimulation.

Lemma E.10. X-prebisimulations are closed under unions.

Corollary E.11. The union $\sim_{X}^{\vee, \otimes}$ of all $X$-prebisimulations over $X^{2}$, called $X$-prebisimilarity, is an $X$-prebisimulation.

Proposition E.12. Calling X-PreBisim $(X)$ and X-Bisim $(X)$ the posets of $X$-prebisimulations and $X$-bisimulations over $X^{2}$, the monotone maps

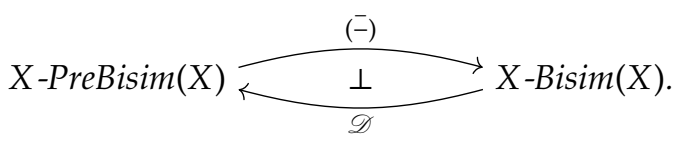

form a Galois connection over the one of Corollary E.6.

Proof. The only non-trivial part lies in showing that if $R$ is an $X$-prebisimulation over $X$ then $\bar{R} \hookrightarrow X^{2}$ is an $X$ bisimulation. We already know from Lemma E.1 that it is a bisimulation, and furthermore $\mathscr{D}(\bar{R})$ is a $\mathscr{D}(X)$-module through the following composite

$$
\mathscr{D}(\bar{R}) \otimes \mathscr{D}(X) \cong \mathscr{D}(\mathscr{M}(R)) \otimes \mathscr{D}(X)=R \otimes \mathscr{D}(X) \rightarrow R,
$$

where the first isomorphism follows from Lemma E.2 and the well-known fact [22, Corollary 2.1.15] that in a cofibrantly generated factorisation system all members of the left class 
are retracts of transfinite composites of pushouts of generators, hence if some functor maps all generators to isomorphisms, then it in fact does so for the whole left class.

Proof of Proposition 5.6. As a right adjoint to $\overline{(-)}, \mathscr{D}$ preserves the maximum element.

\section{E.2 Howe closure}

Before attacking the proof of Lemma 5.9, we need the following lemmas.

Lemma E.13. Under the hypotheses of Lemma 5.9, $R^{\bullet}$ is a pointed I-module.

Proof. We need to show that $R^{\bullet} \otimes I \rightarrow \mathbf{Z}^{2}$ factors through $R^{\bullet} \rightarrow \mathbf{Z}^{2}$. Because tensor product preserves colimits on the left, this reduces to finding a cocone $\left(R_{n} \otimes I \rightarrow R^{\bullet}\right)_{n}$ over $\mathbf{Z}^{2}$, which we do by induction on $n$. The base case is trivial by preservation of the initial object by tensor product. Assuming $R_{n} \otimes I \rightarrow R^{\bullet}$ over $\mathbf{Z}^{2}$, by preservation of coproducts and pullbacks by tensor product on the left, we have $R_{n+1} \otimes I \cong I \otimes I+\left(\Sigma_{0}\left(R_{n}\right) \otimes I ; R \otimes I\right)$. But $R$ is a pointed $I$-module by hypothesis, and $I$ is one in any skew-monoidal category, hence we get $R_{n+1} \otimes I \rightarrow I+\Sigma_{0}\left(R_{n} \otimes I\right) ; R$ hence the result by induction hypothesis.

Lemma E.14. Given relations $A, B, C$ over $X$, we have $(A ; B) \otimes$ $C \subseteq(A \otimes C) ;(B \otimes X)$.

Proof. By tensoring the defining pullback of $A ; B$ with $C$ we obtain the back face below.

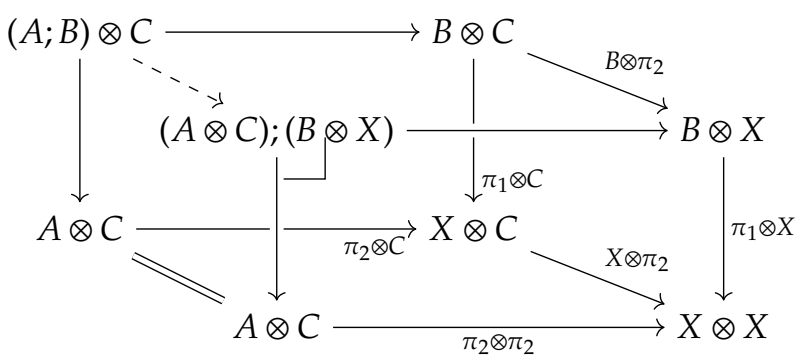

By universal property of pullback, we then get the dashed arrow making all faces commute, which entails the result.

Proof of Lemma 5.9.

(i) We proceed by induction, as $\mathbf{Z}=\operatorname{colim}_{n} \mathbf{Z}_{n}(0)$, where $\mathbf{Z}_{0}=0$ and $\mathbf{Z}_{n+1}=I+\Sigma_{0}\left(\mathbf{Z}_{n}\right)$. The base case is trivial, and assuming $\mathbf{Z}_{n} \rightarrow \mathbf{Z} \rightarrow \mathbf{Z}^{2}$ factors through $R_{n} \rightarrow \mathbf{Z}^{2}$, we have that $\Sigma_{0}\left(R_{n}\right) \rightarrow \mathbf{Z}^{2}$ factors through $\Sigma_{0}\left(R_{n}\right) ; R \rightarrow \mathbf{Z}^{2}$ by reflexivity, hence so does $\Sigma_{0}\left(\mathbf{Z}_{n}\right) \rightarrow$ $\mathbf{Z}^{2}$, which easily entails that $\mathbf{Z}_{n+1} \rightarrow \mathbf{Z}^{2}$ factors through $R_{n+1} \rightarrow \mathbf{Z}^{2}$, as desired.

(ii) By commutation of filtered colimits with finite limits (in presheaf categories), we have $R^{\bullet} ; R \cong \operatorname{colim}_{n}\left(R_{n} ; R\right)$, which reduces the problem to finding a cocone $\left(R_{n} ; R \hookrightarrow\right.$ $\left.R^{\bullet}\right)_{n}$, for then we conclude by universal property of the colimit. We proceed by induction. The base case is trivial, and assuming $R_{n} ; R \hookrightarrow R^{\bullet}$ over $\mathbf{Z}^{2}$, since coproducts commute with connected colimits in general hence pullbacks in particular, we have

$$
R_{n+1} ; R \cong I ; R+\Sigma_{0}\left(R_{n}\right) ; R ; R .
$$

Because $I ; R \hookrightarrow R_{1}$ and by transitivity of $R$, this embeds into $R_{n}+\Sigma_{0}\left(R_{n}\right) ; R$, which indeed embeds into $R^{\bullet}$ by construction, hence the result.

(iii) Because $\Sigma_{0}$ is finitary and filtered limits commute with finite limits in general hence pullbacks in particular (in presheaf categories), we have

$$
\Sigma_{0}\left(R^{\bullet}\right) ; R \cong \operatorname{colim}_{n} \Sigma_{0}\left(R_{n}\right) ; R .
$$

Hence, because $\Sigma_{0}\left(R_{n}\right) ; R \hookrightarrow R^{\bullet}$ for all $n$, we have a cocone to $\left(\Sigma_{0}\left(R_{n}\right) ; R \hookrightarrow R^{\bullet}\right)_{n}$, hence by universal property of the colimit $\Sigma_{0}\left(R^{\bullet}\right) ; R \hookrightarrow R^{\bullet}$, hence by reflexivity of $R$ also $\Sigma_{0}\left(R^{\bullet}\right) \hookrightarrow R^{\bullet}$, as desired.

(iv) Finally, tensor product preserves colimits on the left, so we have $R^{\bullet} \otimes R^{\bullet} \cong \operatorname{colim}_{n}\left(R_{n} \otimes R^{\bullet}\right)$, which reduces the problem to finding a cocone $\left(R_{n} \otimes R^{\bullet} \hookrightarrow R^{\bullet}\right)_{n}$ over $\mathbf{Z}^{2}$, for we then conclude by universal property. We proceed by induction. The base case follows by preservation of colimits, hence of the initial object, on the left by tensor products, and assuming $R_{n} \otimes R^{\bullet} \hookrightarrow R^{\bullet}$, by Lemma E.14, we have

$$
R_{n+1} \otimes R^{\bullet} \subseteq I \otimes R^{\bullet}+\left(\Sigma_{0}\left(R_{n}\right) \otimes R^{\bullet}\right) ;(R \otimes \mathbf{Z}),
$$

hence may proceed componentwise. We clearly have $\lambda_{R^{\bullet}}: I \otimes R^{\bullet} \rightarrow R^{\bullet}$. Furthermore, by Lemma E.13, $R^{\bullet}$ is a pointed $I$-module, so by structural strength we have

$$
\Sigma_{0}\left(R_{n}\right) \otimes R^{\bullet} \rightarrow \Sigma_{0}\left(R_{n} \otimes R^{\bullet}\right) \rightarrow \Sigma_{0}\left(R^{\bullet}\right),
$$

the second arrow by induction hypothesis. Finally, $R$ is substitution-closed, so $R \otimes \mathbf{Z} \subseteq R$, hence we get $\left(\Sigma_{0}\left(R_{n}\right) \otimes R^{\bullet}\right) ;(R \otimes \mathbf{Z}) \rightarrow \Sigma_{0}\left(R^{\bullet}\right) ; R \rightarrow R^{\bullet}$ by the previous points, which concludes the proof.

Lemma E.15. If $R \sim_{l} R^{+}$, then $R^{+} \sim_{l} R^{+}$.

Proof of Lemma 5.13. $R^{\bullet+}$ is transitive by construction, and reflexive because $R^{\bullet}$ is by Lemma 5.9(i). For symmetry, it suffices to show that $R^{\bullet}$ is included in the converse $R^{\bullet+\dagger}$ of its transitive closure. This in turn follows by induction from Lemma E.15:

- Clearly, $\mathbf{Z}$ and $R$ are included in $R^{\bullet++}$.

- It is then enough to show that if $R_{n}$ is included in $R^{\bullet++}$, then so is $\Sigma_{0}\left(R_{n}\right) ; R$. And indeed we have

$$
\Sigma_{0}\left(R_{n}\right) \subseteq \Sigma_{0}\left(R^{\bullet+\dagger}\right)=\left(\Sigma_{0} R^{\bullet+}\right)^{\dagger} \subseteq R^{\bullet+\dagger}
$$

(using Lemma E.15 for the last inclusion) and so

$$
\Sigma_{0}\left(R_{n}\right) ; R \subseteq R^{\bullet+\dagger} ; R^{\bullet+\dagger} \subseteq R^{\bullet+\dagger},
$$

as desired. 


\section{E.3 Cellularity (proofs)}

Although Theorem 5.19 is mostly proved in $§ 5.4$, we give a bit more detail in this section. First, in $§ E .3 .1$, we sketch a proof of Lemma 5.16. The following three sections provide a bit more detail on particular points. We first prove that the morphism $P \rightarrow R^{\bullet}$ factors through some $\Sigma_{0}\left(R^{\bullet}\right) ; R$. We furthermore explain how (33) decomposes into (34). We then give more detail about how the various implicit coercions are handled, and how generics transfer across adjunctions. Finally, Section E.3.5 provides a proof of Lemma 5.20.

\section{E.3.1 Proof sketch for Lemma 5.16.}

Notation E.16. For any functor $F$, let $\rightarrow$ denote free morphisms, i.e., morphisms in the image of $F$. Commutation of a diagram made of such arrows means commutation before applying $F$.

Notation E.17. For conciseness, instead of assuming the induction hypothesis for $n-1$ and proving the result for $n$, we assume it for $n$ and prove it for $n+1$.

By Lemma 4.4, an opcartesian lifting $\Sigma_{1} \rightarrow \check{\Sigma}_{1}$ is epi at any $L$, so we further factor the given morphism through $\Sigma_{1}\left(\check{\Sigma}_{1}^{n}\left(0^{\circledast}\right)\right)$. Moreover, by construction of $R^{\bullet}$, the top morphism factors through $\Sigma_{0}\left(R^{\bullet}\right) ; R \rightarrow R^{\bullet}$. By definition of sequential composition, we thus get a square

$$
\begin{array}{cc}
P \longrightarrow \Sigma_{0}\left(R^{\bullet}\right) \\
\downarrow \\
\stackrel{\downarrow}{L} \longrightarrow \mathbf{Z},
\end{array}
$$

which we will complete as in (17), with $R^{\prime}=\mathscr{T}_{0}\left(R^{\bullet}\right)$. By weak compositionality of $\mathbf{Z}$, familiality of $\Sigma_{0}$ and $\Sigma_{1}$, and cartesianness of $\Sigma_{0} \rightarrow \Sigma_{1}$ (which follows from Theorem 2.19), it factors as below.

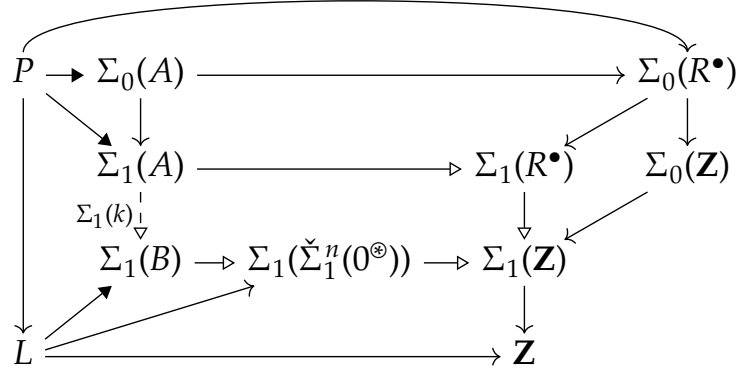

By generic factorisation of the composite $Q \rightarrow L \rightarrow \Sigma_{1}(\mathbf{Z})$ (though through $\mathscr{T}_{0}$ instead of $\Sigma_{0}$ ), we obtain precisely the diagram (19, left) - with $n$ instead of $n-1$ because of different conventions - which we complete by hypothesis. Let us now use this to complete the square (33) as desired. The obtained morphism $L \rightarrow \mathbf{Z}$ yields a square

$$
\begin{aligned}
& P \rightarrow R \\
& \downarrow \\
& L
\end{aligned} \begin{aligned}
& \downarrow \\
& \mathbf{Z},
\end{aligned}
$$

which we may complete because $R$ is a prebisimulation. Finally, we take the pairing of the obtained morphisms $Q \rightarrow$ $\mathscr{T}_{0}\left(R^{\bullet}\right)$ and $Q \rightarrow R$ to get a morphism $Q \rightarrow \mathscr{T}_{0}\left(R^{\bullet}\right) ; R$, hence $Q \rightarrow R^{\bullet}$ by Corollary 5.10, which completes the original diagram as desired.

E.3.2 Factorisation of $P \rightarrow R^{\bullet}$. The only way for $P \rightarrow$ $R^{\bullet}$ not to factor through some $\Sigma_{0}\left(R^{\bullet}\right) ; R$ is to factor through $I$. But in this case we would have a commuting square

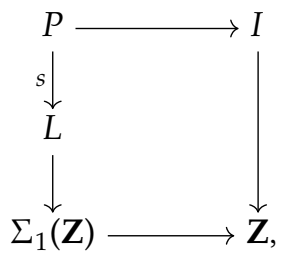

which because $\left(\Sigma_{0}, \Sigma_{1}\right)$ is layered refactors as

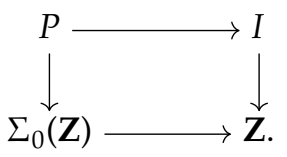

But the cospan $I \rightarrow \mathbf{Z} \leftarrow \Sigma_{0}(\mathbf{Z})$ is a coproduct diagram at each state type $P$, hence the pullback is empty because coproducts are disjoint in presheaf categories, which renders impossible the existence of any square of the form (35).

E.3.3 Decomposition into (34). Let us now explain why the square (33) decomposes as (34). Letting $\mathbf{Z}_{n}=\check{\Sigma}_{1}^{n}\left(0^{\circledast}\right)$, the given square factors as the exterior of

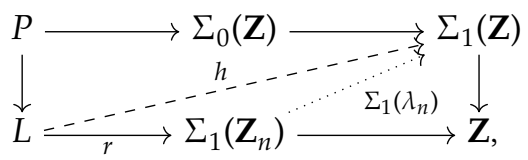

so we get a dashed $k$ as shown by weak compositionality. Furthermore, the triangle below right commutes by construction, so because $\Sigma_{1}(\mathbf{Z}) \rightarrow \mathbf{Z}$ is monic at each $L$, we get $\Sigma_{1}\left(\lambda_{n}\right) \circ r=h$. The rest is easy by familiality of $\Sigma_{1}$ and $\Sigma_{0}$ and preservation of generics by $\Sigma_{0} \rightarrow \Sigma_{1}$.

E.3.4 Coercions and transfer of generics across adjunctions. First of all, the given top morphism in (34) has in fact type $\mathscr{M} P \rightarrow \mathscr{M} \Sigma_{0} R^{\bullet}$. By full faithfulness of $\mathscr{M}$, we find its unique antecedent by $\mathscr{M}$, say $f: P \rightarrow \Sigma_{0} R^{\bullet}$. We factor $f$ as $P \stackrel{\xi}{\rightarrow} \Sigma_{0} A \stackrel{\Sigma_{0} f^{\prime}}{\longrightarrow} \Sigma_{0} R^{\bullet}$ with $\xi$ generic. Our abuse of notation is harmless, though, because we have:

Lemma E.18. For any functor $F: \mathscr{A} \rightarrow \mathscr{B}$, any fully-faithful functor $G: \mathscr{B} \rightarrow \mathscr{E}$ preserves $F$-generics, in the sense that if $f: D \rightarrow F C$ is F-generic, then $G(f): G(D) \rightarrow G F C$ is GFgeneric.

Furthermore, the alleged naturality square 


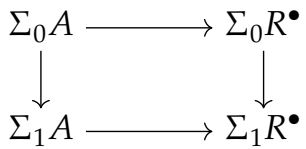

should in fact be refined as follows, the natural transformation actually being

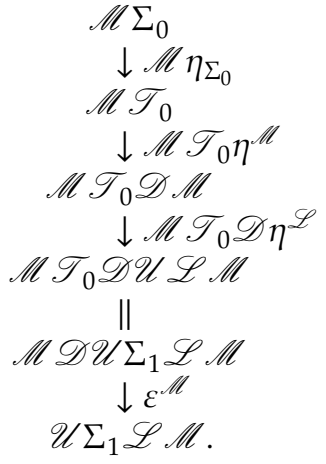

A first problem is that we reach $\mathscr{U} \Sigma_{1} \mathscr{L} \mathscr{M} R^{\bullet}$, so we need to postcompose the right-hand morphism by

$$
\mathscr{U} \Sigma_{1} \mathscr{L} \mathscr{M} R \stackrel{\mathscr{U} \Sigma_{1} \mathscr{L} \pi_{1}}{\longrightarrow} \mathscr{U} \Sigma_{1} \mathscr{L} \mathscr{U} \mathbf{Z} \stackrel{\mathscr{U} \Sigma_{1} \varepsilon_{\mathbf{Z}}^{\mathscr{Z}}}{\longrightarrow} \mathscr{U} \Sigma_{1} \mathbf{Z},
$$

still maintining equality with the top right path of the diagram.

It remains to show that the obtained composite $\mathscr{M} P \rightarrow$ $\mathscr{U} \Sigma_{1} \mathscr{L} \mathscr{M} A$ is $\mathscr{U} \Sigma_{1}$-generic (the rest goes roughly as announced). That postcomposition with $\eta_{\Sigma_{0}}$ preserves genericness follows from Theorem 2.19. The rest follows from various applications of the following two lemmas.

Lemma E.19. Given any adjunction

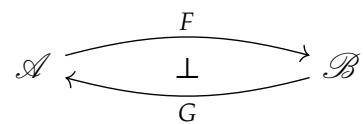

and functor $H: \mathscr{X} \rightarrow \mathscr{B}$, if a morphism $\xi: A \rightarrow G H X$ is $G H$-generic, then its transpose $\check{\xi}: F A \rightarrow H X$ is $H$-generic.

Proof. By transposition, liftings for any square of the form below left are exactly the same as liftings for the corresponding square below right, and conversely.

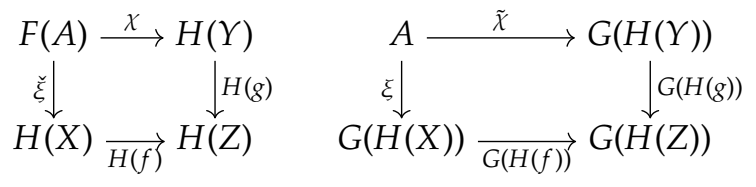

Lemma E.20. Given any adjunction

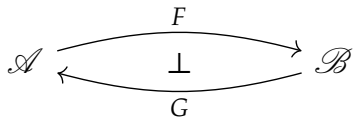

and functor $H: \mathscr{X} \rightarrow \mathscr{A}$, if a morphism $\xi: A \rightarrow H X$ is $H$-generic, then the composite

$$
A \stackrel{\xi}{\rightarrow} H X \stackrel{H\left(\eta_{X}\right)}{\longrightarrow} H G F X
$$

is HG-generic.
Proof. Consider any diagram as the exterior of

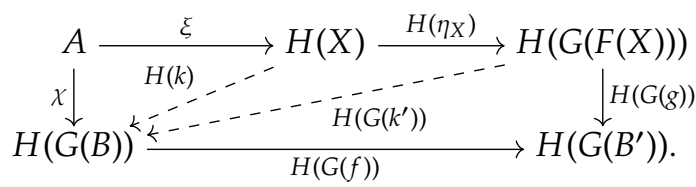

By genericness of $\xi$, we first find a unique lifting $k$. By universal property of $\eta_{X}$, we then find a unique $k^{\prime}$ as shown, such that $G\left(k^{\prime}\right) \circ \eta_{X}=k$, which by uniqueness further satisfies $f \circ k^{\prime}=g$, as desired.

E.3.5 Proof of Lemma 5.20. The only non-trivial point is composition: consider any presimulation $R$, and start from the solid part of the diagram below, where $F$ is the composite, obtained by pushout.

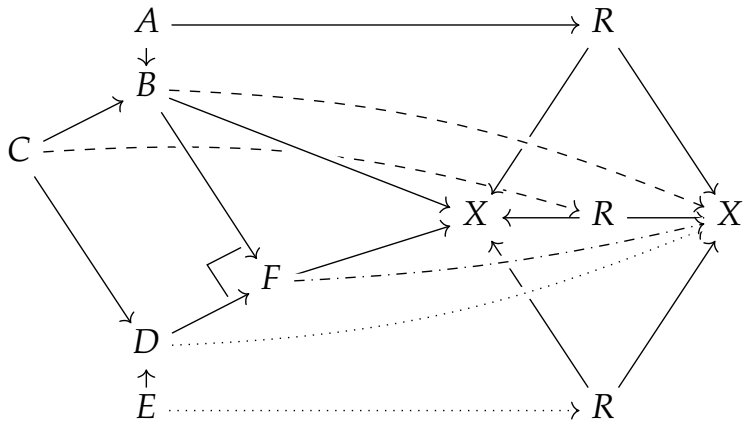

By hypothesis, we obtained first the dashed, and then, inductively, the dotted arrows. By universal property of pushout, we finally obtained the dash/dotted one, as desired.

Here is a perhaps more geometrical perspective on the same argument, with the same graphical conventions, bearing in mind that only two out of four 'squares' in the left and right faces are proper (commuting) squares.

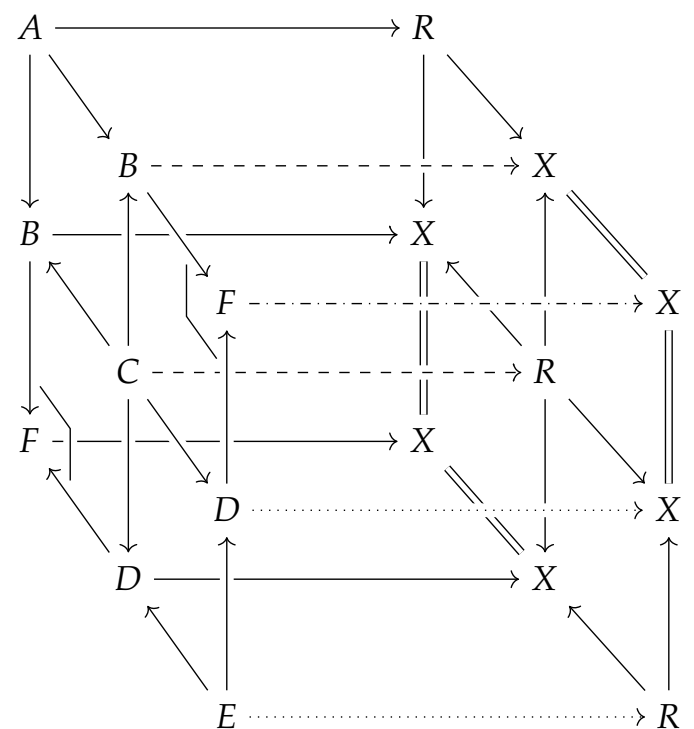




\section{F Conclusion and perspectives}

The additional coherence condition alluded to in $\S 6$ consists in demanding $I$-modules $(A, r, e)$ to further make the following diagram commute.

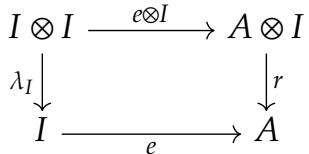

